\title{
Interplay between brassinosteroids and TORC signaling in Arabidopsis revealed by integrated multi-dimensional analysis
}

\author{
Christian Montes ${ }^{1}$, Ching-Yi Liao ${ }^{2}$, Trevor M Nolan ${ }^{2,3}$, Gaoyuan Song ${ }^{1}$, Natalie M Clark ${ }^{1}$, Hongqing \\ Guo $^{2}$, Diane C Bassham², Yanhai Yin ${ }^{2}$, and Justin W Walley ${ }^{1, \bigotimes}$ \\ ${ }^{1}$ Department of Plant Pathology and Microbiology, lowa State University, Ames, IA 50011 \\ ${ }^{2}$ Department of Genetics, Developmental, and Cell Biology, lowa State University, Ames, IA 50011 \\ ${ }^{3}$ Department of Biology, Duke University, Durham, NC 27708, USA
}

\begin{abstract}
Brassinosteroids (BR) and Target of Rapamycin Complex (TORC) are two major processes coordinating plant growth and stress responses. BRs function through a signaling pathway to extensively regulate gene expression and TORC is known to regulate translation and autophagy. Recent studies revealed that these two pathways crosstalk, but a system-wide view of their interplay is still missing. Thus, we performed transcriptome, proteome, and phosphoproteome profiling of Arabidopsis mutants with altered levels of either BIN2 or RAPTOR1B, two key players in BR and TORC signaling, respectively. We found that perturbation of BIN2 or RAPTOR1B levels affects a common set of gene-products involved in growth and stress responses. Additionally, we performed Multiplexed Assay for Kinase Specificity (MAKS), which provided a system-wide view of direct BIN2 substrates. Furthermore, phosphoproteomic data was used to reconstruct a kinase-signaling network and to identify novel proteins dependent on $B R$ and/or TORC signaling pathways. Loss of function mutants of many of these proteins led to an altered BR response and/or modulated autophagy activity. Altogether, these results provide genome-wide evidence for crosstalk between BR and TORC signaling and established a kinase signaling network that defines the molecular mechanisms of BR and TORC interactions in the regulation of plant growth/stress balance.
\end{abstract}

Autophagy | BIN2 | Brassinosteroid | Kinase | Multi-omics | Phosphorylation | Target of Rapamycin

Correspondence: Justin Walley (jwalley@iastate.edu)

\section{Main}

When plants respond to stress, specific molecular and cellular processes are triggered, and growth is often compromised. Therefore, well-coordinated crosstalk of different signaling pathways is fundamental for a successful response (Huot et al., 2014; Verma et al., 2016). The growth-promoting hormone brassinosteroid $(B R)$ is critical for this balance. Plants with perturbed BR biosynthesis or signaling exhibit altered growth and response to stresses (Clouse et al., 1996; Li et al., 1996;
Guo et al., 2013; Nolan et al., 2017a, 2017b; Gruszka, 2018; Ye et al., 2019; Nolan et al., 2020). The Glycogen Synthase Kinase 3 (GSK3)-like kinase BIN2 is a pivotal negative regulator of $\mathrm{BR}$ signaling ( $\mathrm{Li}$ and Nam, 2002; Kim et al., 2009). In the absence of BRs, BIN2 phosphorylates the BES1/BZR1 family of transcription factors (TFs), reducing their protein levels, DNA binding, and promoting cytoplasmic sequestration by $14-3-3$ proteins, thereby preventing the activation of downstream BR response genes (Ryu et al., 2007; Gampala et al., 2007; Ryu et al., 2010; Yin et al., 2002). Besides regulating BES1 and BZR1, increasing evidence positions BIN2 as a hub for stress and growth balance regulation (Cai et al., 2014; Cho et al., 2014; Hu and Yu, 2014; Youn and Kim, 2015; Jiang et al., 2019; Ye et al., 2019; Li et al., 2020a; Nolan et al., 2020; Li et al., 2020b).

In Arabidopsis, the target of rapamycin regulatory complex (TORC) is a vital regulator integrating nutrient and energy sensing into cell proliferation and growth (Xiong and Sheen, 2014; Fu et al., 2020). The complex is comprised of TOR kinase, LST8, and RAPTOR. RAPTOR interacts with and recruits substrates to the complex for phosphorylation by TOR (Hara et al., 2002; Yang et al., 2013). Two RAPTOR homologs, RAPTOR1A and $R A P T O R 1 B$, have been found in Arabidopsis with RAPTOR1B being the predominantly expressed copy (Anderson et al., 2005; Deprost et al., 2005). Activation of TORC signaling induces the expression of ribosomal proteins, increases protein translation, stimulates photosynthesis, and upregulates (transcriptionally and translationally) plant growth-promoting genes (Ren et al., 2012; Xiong et al., 2013; Dong et al., 2015; Van Leene et al., 2019; Scarpin et al., 2020). Conversely, TORC actively represses autophagy, a central recycling system of cytoplasmic components and whose regulation is essential for rerouting nutrients and other raw materials when needed for plant growth, development, or stress responses (Noda and Ohsumi, 1998; Pu et al., 2017; Marshall and Vierstra, 2018).

When plants encounter stress, autophagy is often triggered, and growth-promoting pathways such as BR or TORC signaling need to be dampened (Nolan et al., 2017a; Liao and Bassham, 2020). To enable this balanced regulation of plant growth and stress responses, hormonal 
pathways such as auxin ( $\mathrm{Li}$ et al., 2017; Schepetilnikov et al., 2017) and BRs (Zhang et al., 2016; Vleesschauwer et al., 2018) can influence or be affected by TORC activity. Increasing evidence points towards TORC-regulated autophagy as a crucial interaction point between BRs and TORC signaling when controlling this balance. For example, activation of TORC signaling promotes $B R$ response by stabilizing BZR1, likely preventing its autophagy-driven degradation (Zhang et al., 2016). Additionally, BIN2 knock-down lines exhibit reduced sensitivity to TOR inhibitors AZD8055 (AZD) and KU63794 (Xiong et al., 2017). Furthermore, S6K2 can phosphorylate BIN2 in a TOR-dependent manner. However, the mechanism and biological implications of this interaction are not clear. Moreover, BIN2 has been shown to phosphorylate ubiquitin receptor DSK2 to facilitate its interaction with ATG8 and promote BES1 degradation via selective autophagy (Nolan et al., 2017b).

BIN2 and TORC regulate plant response to environmental changes via phosphorylation, exerting molecular changes at many different levels (i.e., changes in gene transcription or protein activity) (Guo et al., 2013; Bozhkov, 2018; Van Leene et al., 2019; Liao and Bassham, 2020; Nolan et al., 2020). Therefore, understanding the molecular connection between BR and TORC signaling across different levels of gene expression is necessary to unravel the interplay between these pathways. Furthermore, despite BIN2 being intensively studied, proteome-wide identification of BIN2 substrates is lacking. Here, we present a comprehensive multi-omic profiling detailing transcriptome, proteome, and phosphoproteome changes that occur in mutants with altered levels of BIN2 or the TORC subunit RAPTOR1B. We complement these global in vivo profiles with proteomewide identification of direct BIN2 substrates using a Multiplexed Assay for Kinase Specificity (MAKS). A significant overlap was found in the transcripts, proteins, and phosphosites whose accumulation is dependent on BIN2 and RAPTOR1B. We reconstructed a kinase-signaling network and used it to identify novel genes whose mutant lines showed either altered growth in response to BR and/or levels of autophagy. Together, these studies further our understanding of the dynamic interplay between BR and TORC signaling.

\section{Results}

Comprehensive multi-omics profiling of bin2 and raptor1b mutants. We designed a multi-omics experiment to identify novel components dependent on BR and/or TORC signaling. We performed transcriptome, proteome, and phosphoproteome profiling on rosette leaves of 20-day old wild-type (WT), bin2D (gain-of-function), bin2T (bin2 bil1 bil2 triple loss of function mutant), and raptor $1 b$ plants. We quantified transcript levels using 3' QuantSeq and measured protein abundance and phosphorylation state using two-dimensional liquid chromatography-tandem mass spectrometry (2D-LC-MS/MS) on Tandem Mass Tag
(TMT) labeled peptides (McAlister et al., 2012; Hogrebe et al., 2018; Song et al., 2018a) (Fig. 1a). From these samples, we detected 23,975 transcripts, 11,183 proteins, and up to 27,887 phosphosites from 5,675 phosphoproteins (Fig. 1b and Supplementary Data Set 1).

We found 5,653 transcripts and 4,001 proteins that were differentially expressed (DE) in at least one mutant when compared against WT (Fig. 1c and Supplementary Fig. 1a and 2a). Gene ontology (GO) analysis of $D E$ transcripts and proteins in bin2D, bin2 $T$, and raptor1b mutants showed enrichment of many terms from similar processes including growth, hormones, stimuli sensing, and stress (Supplementary Figs. 1 and 2). These results suggest that the biological processes being affected at both protein and transcript level remain closely linked. Furthermore, the enriched terms among bin2 and raptor1b DE transcripts and proteins are consistent with their known roles in growth/stress balance and hormonal crosstalk (Supplementary Data Set 2 and 3).

Our phosphoproteomics analysis identified 4,153 differentially phosphorylated sites in at least one mutant (Fig. 1c and Supplementary Fig. 3a). GO analysis for potential BIN2 target proteins (i.e., those with increased phosphorylation in bin2D or decrease phosphorylation in bin2T) revealed enrichment of terms related to plant growth and development, as well as response to stress and defense, processes in accordance with known BR functions. In addition, response to BR, abscisic acid, and auxin terms were also significant, highlighting once more the close relationship between BIN2 activity and these hormones. Transcriptional regulation-related terms were significantly enriched in the bin2D dataset, consistent with BIN2's well documented regulatory activity upon TFs (Supplementary Fig. 3b,c and Supplementary Data Set 4). Finally, we assessed GO enrichment for proteins with decreased phosphorylation in raptor $1 b$. We found that most of the enriched terms were related to growth, autophagy, starvation, auxin, and BR response. This is consistent with the known biological role of RAPTOR and suggests a cross-regulation between BR and TORC pathways via phospho-signaling (Supplementary Fig. 3d and Supplementary Data Set 4).

BIN2 and RAPTOR1B regulate a core set of common gene-products. Next, we compared the DE transcripts we identified with previously reported transcriptomic data for BR and TORC. We found a significant overlap between the DE transcripts in bin2 mutants and transcripts that respond to brassinolide (BL) treatment (Wang et al., $2014) ; 40.2 \%$ of bin2D $(p=6.14 \mathrm{e}-45)$ and $36.8 \%$ bin2T $(p=2.85 \mathrm{e}-24)$ responded to $\mathrm{BL}$ treatment. Interestingly, $30.9 \%$ of raptor $1 b$ DE transcripts $(p=6.55 \mathrm{e}-22)$ overlapped with $\mathrm{BL}$ responsive transcripts (Supplementary Fig. 4a). We also compared our data to the transcriptome 
a

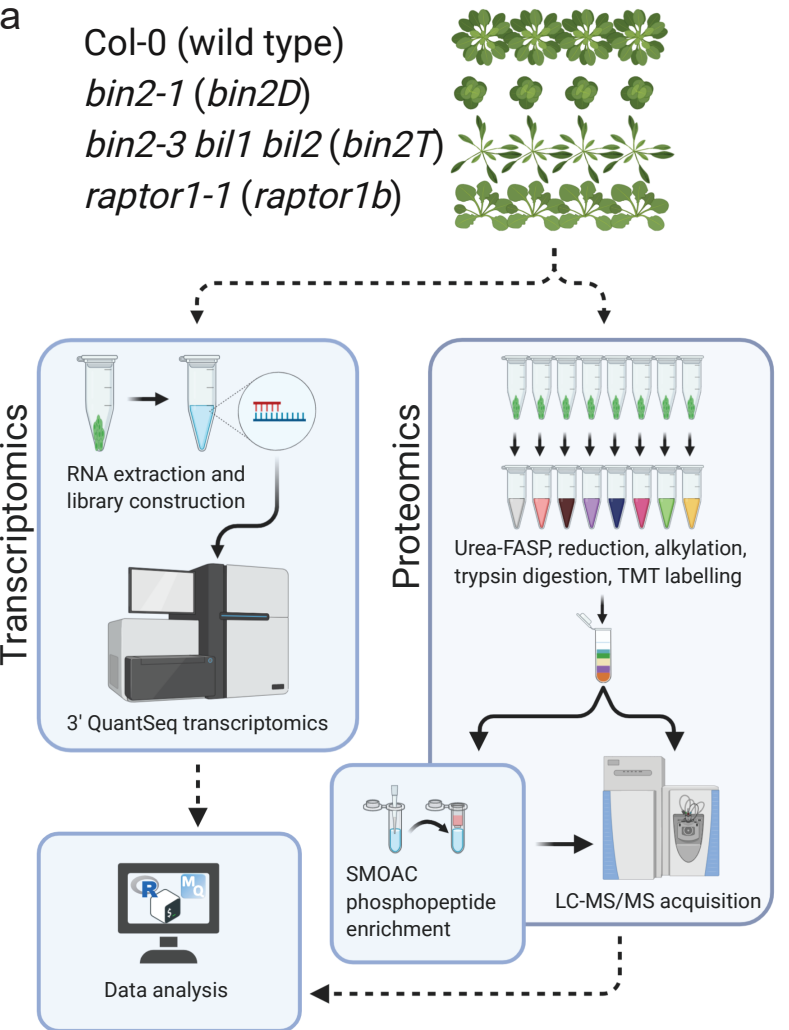

b

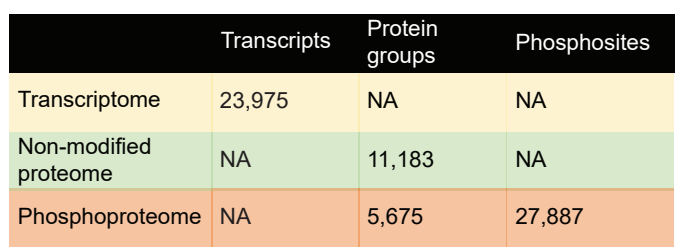

C

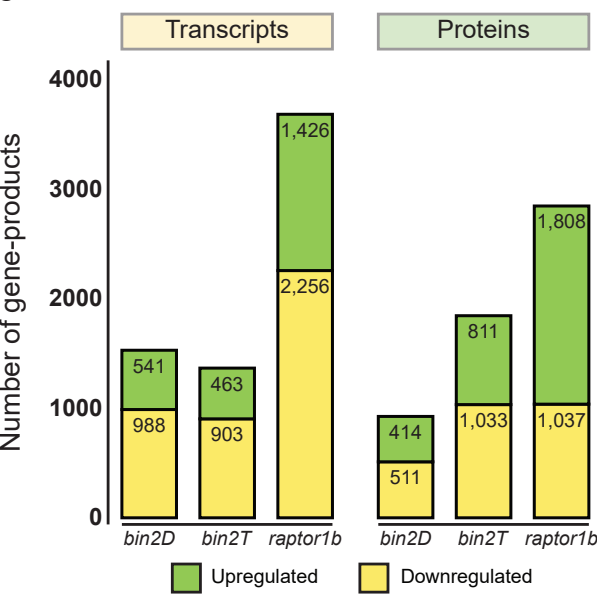

Phosphosites

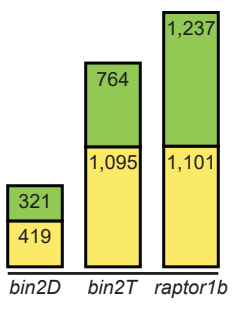

Fig. 1. Experimental design, workflow, and data overview. a, Schematic representation of the multi-omics processing pipeline for bin2 and raptor $1 b$ mutants. b, number of total detected transcripts, proteins, phosphoproteins, and phosphosites. c, differentially expressed transcripts, proteins, and phosphorylated amino acids for each analyzed mutant compared to WT.

profile of 10-day-old Arabidopsis seedlings treated with mock or AZD8055 (AZD), a specific TOR kinase inhibitor (Dong et al., 2015). We observed a significant overlap ( $p=1.03 \mathrm{e}-15)$ between the mis-expressed transcripts in raptor $1 b$ and transcripts DE in AZD treated plants. Similar to the overlap between raptor $1 b$ and BL treatment, AZD responsive transcripts exhibited a significant overlap with transcripts DE in both bin2 mutants. Specifically, 25.5\% of transcripts DE in bin2D ( $p=5.85 \mathrm{e}-38)$ and $26.2 \%$ of transcripts DE in bin2T $(p=1.18 \mathrm{e}-35)$ were also AZD responsive (Supplementary Figure $4 b$ ).

The reciprocal enrichment of BL-responsive transcripts in raptor $1 b$ and AZD (i.e., TORC) responsive transcripts in bin2 mutants prompted us to investigate whether there are common gene-products being regulated by both BIN2 and RAPTOR1B. We found significant overlaps in DE geneproducts between raptor $1 b$ and bin2D as well as between raptor1b and bin2 $T$ mutants, at all three levels (transcriptome, proteome, and phosphoproteome). For our transcriptomic data we found that $25.3 \%$ of genes $D E$ in the bin2D mutant $(p=2.08 \mathrm{e}-15)$ were also $\mathrm{DE}$ in the raptor $1 b$ mutant and $30.0 \%$ of genes DE in bin2T $(p=2.01 \mathrm{e}$ $30)$ were also DE in raptor $1 b$. Interestingly, from our proteomic analyses we found that $55.8 \%$ of proteins $D E$ in bin2D $(p=1.02 \mathrm{e}-24)$ and $50.6 \%$ of those DE in bin2T $(p=$ $3.97 e-26)$ were also DE in the raptor1b mutant. Finally, we found that $26.7 \%$ of bin2D DE phosphosites $(p=2.04 \mathrm{e}-16)$ and $21.9 \%$ of bin2T DE phosphosites ( $p=8.64 \mathrm{e}-16)$ were DE in raptor1b (Fig. 2a). Since we found a significant overlap between our raptor1b mutant and both bin2 mutants, we next investigated the biological processes being jointly affected. GO analysis on the proteins $D E$ in both raptor1b and bin2D or bin2T mutants showed significant enrichment of stress terms (response to toxic substance, response to heat, response to cold, response to oxidative stress) and growth-related processes (photosynthesis, glucose metabolic process, ribosome assembly and translation) (Fig. 2b and Supplementary Data Set 5). These results reveal that BIN2 and RAPTOR1B affect a common core set of gene-products. Additionally, GO analysis suggest an involvement of these genes in processes related to growth and stress responses.

\section{Phosphoproteomic analysis of bin2 mutants shows} enrichment of BIN2 direct targets. Because BIN2 is a kinase, we hypothesized that phosphosites increased in the bin2D gain-of-function mutant or decreased in bin2T may be direct BIN2 substrates. To test this hypothesis, we generated a proteome-wide dataset of BIN2 direct targets using the multiplexed assay for kinase specificity (MAKS) (Brumbaugh et al., 2014; Jayaraman et al., 2017) (See Methods for details). We quantified a total of 10,375 phosphosites accounting for 3,628 phosphoproteins from this assay (Supplementary Data Set 1b). The obtained phosphoproteome was heavily skewed 
a

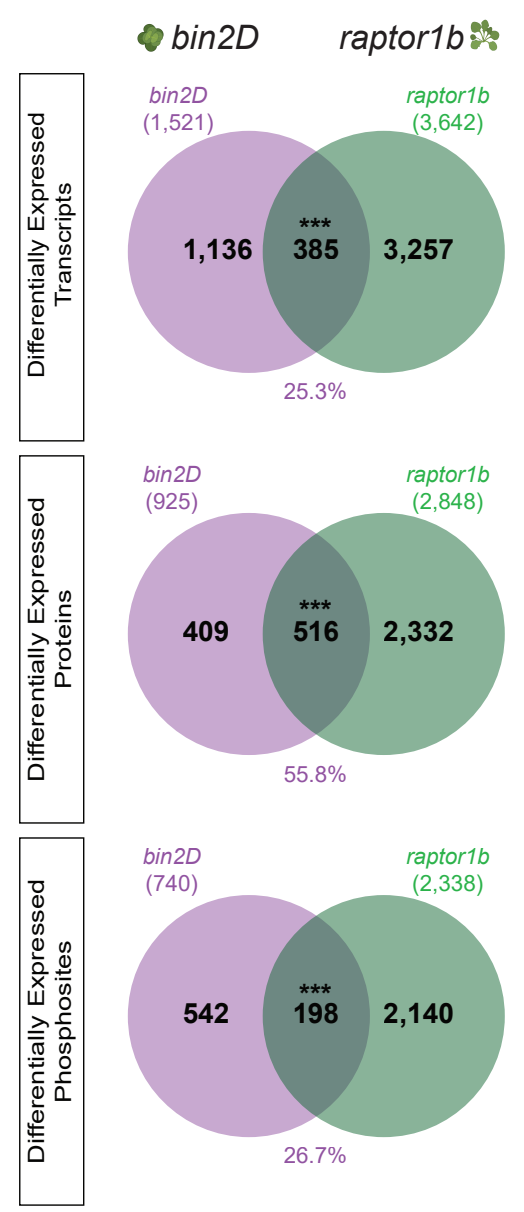

b
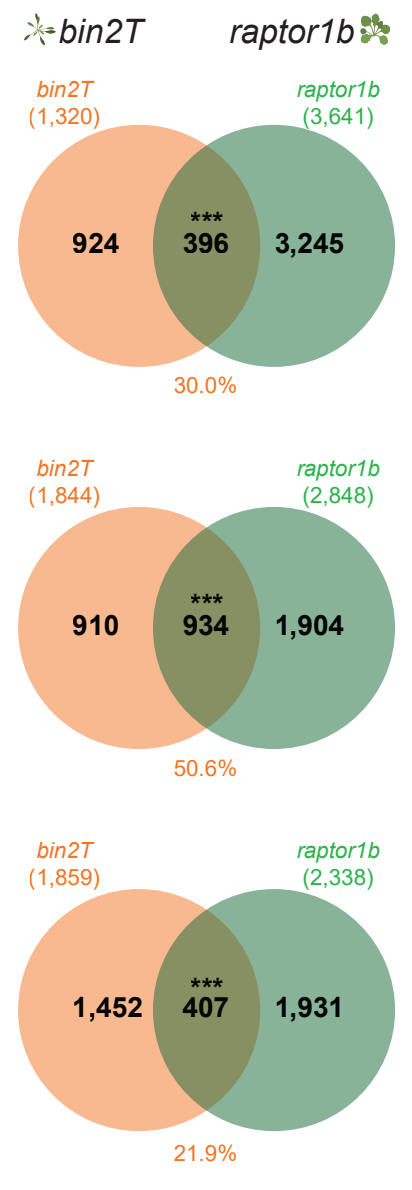

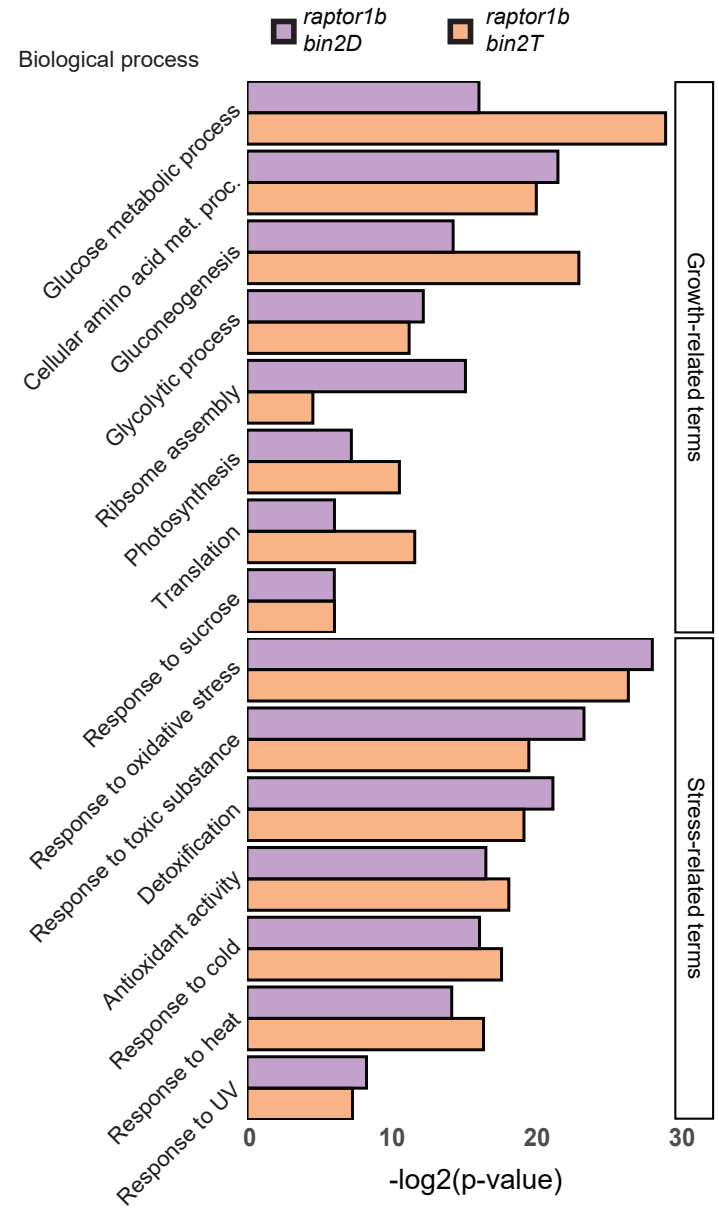

Fig. 2. BIN2 and RAPTOR1b affect a common core set of common gene-products. a, Venn diagram showing overlap of differentially expressed transcripts (upper), proteins (middle), or phosphosites (lower) between raptor1b and bin2D (left) or bin2T (right) mutants. Statistical significance was assayed using hypergeometric test, assessing only gene-products present in both datasets ${ }^{* * *}$ $p<0.001)$. b. Significantly enriched GO biological processes among proteins whose expression is being affected concomitantly by raptor1b and either bin2D (purple) or bin2T (orange). GO significance calculated using Fisher's exact test, significance cutoff was established as q-value $<0.1$.

toward increased phosphosites (Fig. 3a), with 1,343 phosphosites increased following incubation with GSTBIN2 (Supplementary Data Set 1b). Among proteins with increased phosphorylation we observed YDA and BSK1, two known BIN2 targets (Kim et al., 2012; Sreeramulu et al., 2013). To evaluate this set of phosphorylation sites as BIN2 kinase-substrates, we performed motif enrichment analysis and found a significant enrichment of the well-known GSK3 motif "S/T-x-x-x-S/T" (Fiol et al., 1987; Youn and Kim, 2015) among the increased phosphosites ( $p=2.01 \mathrm{e}-25$, Fig. 3b and Supplementary Data Set 6a). Additionally, another highly enriched motif found in the analysis was "S/T-P", which is reported as a motif for GSK3, CDK, and MAPK families (Amanchy et al., 2007; Lin et al., 2015) (Supplementary Data Set 6a). Some previously unreported length variations of the GSK3 motif were also significantly enriched (i.e., S/T-x-x-S/T, $\mathrm{S} / \mathrm{T}-\mathrm{x}-\mathrm{S} / \mathrm{T}$, and S/T-S/T, Supplementary Data Set 6a). These results support the robustness of our BIN2 kinase dataset.
We next assessed the prevalence of BIN2 direct targets present in our profiling of bin2D and bin2T mutants. For this, we first performed motif enrichment analysis on phosphosites either upregulated in bin2D or downregulated in bin2T. A significant enrichment for the GSK3 motif was found in both bin2D $(p=5.05 \mathrm{e}-12)$ and bin2T $(p=8.30 \mathrm{e}-$ 09) phosphosites (Fig. 3b and Supplementary Data Set $6 b, c)$. Next, we looked at the overlap with our BIN2 direct targets identified in the MAKS experiment. For bin2D, $17.0 \%(41 / 241 ; p=6.53 \mathrm{e}-03)$ of the total differentially upregulated phosphosites and $23.6 \%(38 / 161 ; p=2.58 \mathrm{e}-03)$ of the upregulated phosphoproteins were also BIN2 direct targets. For bin2T, $13.8 \%(77 / 558 ; p=5.04 \mathrm{e}-02)$ of the downregulated phosphosites and $23.3 \%(117 / 503 ; p$ $=5.80 \mathrm{e}-06$ ) of the downregulated phosphoproteins were also part of our BIN2 direct substrate list (Fig. 3c and Supplementary Data Set 7). These results indicated that a subset of the BIN2 dependent phosphosites identified by in vivo mutant profiling may be direct BIN2 substrates. 
a

\section{BIN2 whole proteome in-vitro kinase assay}

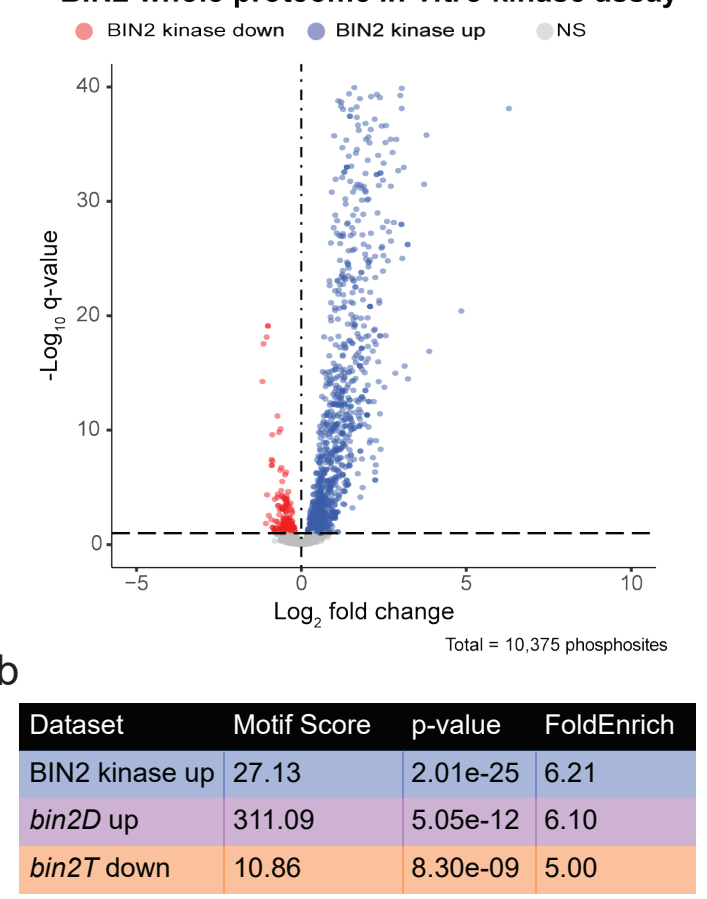

C

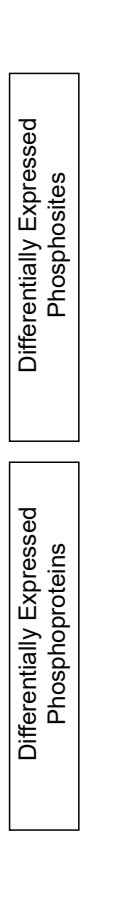

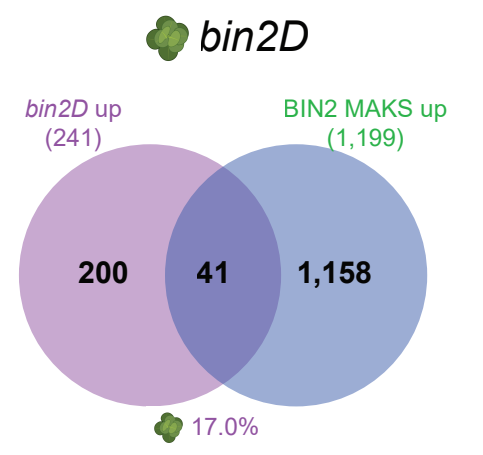
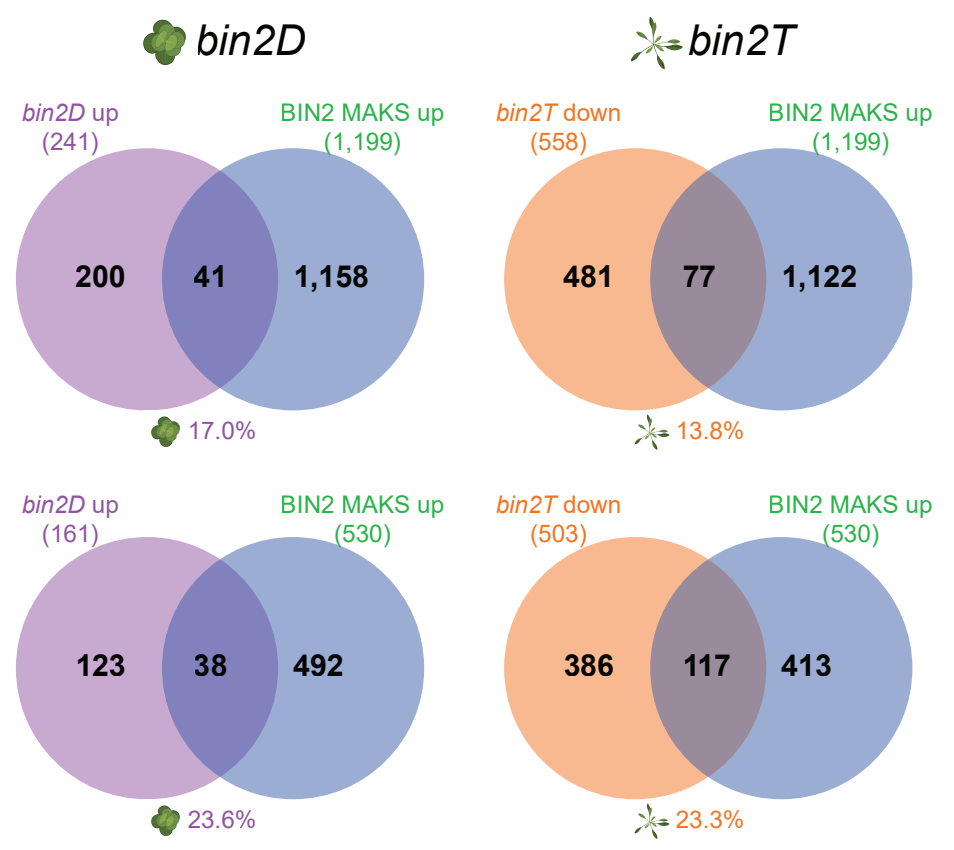

Fig. 3. Phosphoproteomic analysis on BIN2 mutants shows significant enrichment of BIN2 direct targets. a, Volcano plot of phosphorylation sites from a Multiplexed Assay for Kinase Specificity (MAKS) on Arabidopsis leaf protein extracts incubated with recombinant GST or GST-BIN2. Significantly increased phosphosites are colored blue whereas decreased phosphosites are red $(q<0.1)$. b, De novo motif enrichment analysis showed high enrichment for the GSK3 motif on BIN2-related phosphoproteomic datasets. Motif score and FoldEnrich values are calculated by motifeR, while $p$-value was calculated using hypergeometric testing. c, Venn diagrams show overlap between BIN2 direct targets (i.e., those upregulated in BIN2 MAKS) and phosphosites (upper) or phosphoproteins (lower) upregulated in bin2D (left) or downregulated in bin2T (right) mutants. Numbers below each Venn diagram represent the overlapping percent for that mutant (purple $=$ bin $2 D$, orange $=$ bin $2 T$ ). Phosphosite overlaps were calculated using a 20 amino acid window, centered on the differentially regulated phosphosite (for details see methods section). Statistical significance was calculated using hypergeometric testing $\left({ }^{*} p<0.05\right.$, $\left.{ }^{* *} p<0.01\right)$.

Kinase-signaling network inference on bin2 and raptor1b mutants. Since both BIN2 and RAPTOR1B (TORC) participate in phosphorylation-based signaling, differentially accumulating phosphosites identified in our mutant profiling maybe direct or indirect targets of BIN2 and/or RAPTOR1B. With this in mind, we reconstructed the molecular relationships of these signaling networks. To do so, we used our data to infer a kinase-signaling network for each mutant (i.e., bin2D, bin2T, and raptor1b). To build these networks, we first inferred the activation state of kinases in our dataset. The activation loop (Aloop) is a well-conserved region inside the kinase domain whose phosphorylation is necessary for kinase activation (Adams, 2003; Ahiri, 2019). Thus, phosphosite intensity level of the A-loop can be used as a proxy for kinase activity quantification (Walley et al., 2013; Beekhof et al., 2019; Schmidlin et al., 2019). First, we performed a wholeproteome Arabidopsis in-silico A-loop prediction and were able to identify the region on 1,360 proteins (Supplementary Data Set 8a). Subsequently, we identified kinases whose A-loop phosphosite intensity was differentially regulated in at least one of the profiled mutants (Supplemen- tary Fig. 5a). We found 27, 21, and 24 kinases exhibiting an altered activation state in the bin2D, bin2T, and raptor $1 b$ mutant, respectively (Supplementary Data Set $8 b$ ). Using this information, we inferred a kinase-signaling network by correlating phosphosite level with kinase activation state (Supplementary Fig. 5b). A network containing 4,138 nodes, representing 33 activated kinases and 2,284 target sites arising from 1,853 possible substrate proteins was obtained (Fig. 4a and Supplementary Data Set 9). To evaluate this kinase-signaling network, predicted BIN2 targets were obtained (i.e., nodes connected by edges directed outward of BIN2), and motif enrichment analysis was performed. As expected, the GSK3 motif was enriched among BIN2 targets $(p=1.96 \mathrm{e}-06)$. Additionally, the MAPK consensus motif P-X-[pS/pT]-P was overrepresented among MPK6 targets $(p=3.56 \mathrm{e}-16)$. Moreover, several variants of the proline-directed phosphorylation motif $[\mathrm{pS} / \mathrm{pT}]-\mathrm{P}$, were significantly enriched among targets of MPK4 $(p<0.001)$, MPK10 $(p<0.001)$, and BIN2 $(p<0.001)$ (Amanchy et al., 2007; Lin et al., 2015; Rayapuram et al., 2021) (Fig. 4b and Supplementary Data Set 10). Furthermore, 66.7\% (6/9) of known BIN2 targets re- 


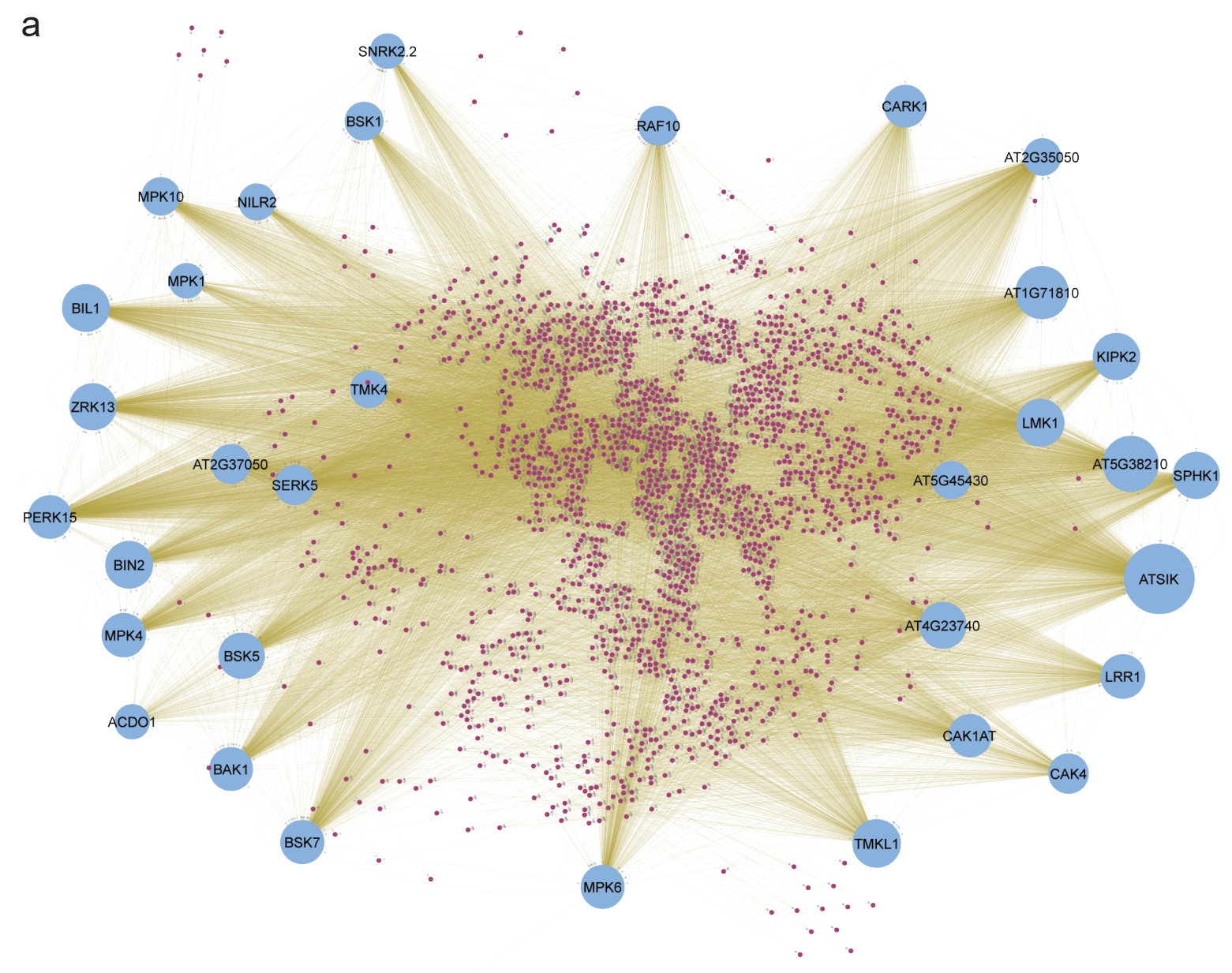

\begin{tabular}{|c|c|c|c|c|c|}
\hline & Nodes & $\begin{array}{l}\text { Regulator } \\
\text { kinases }\end{array}$ & $\begin{array}{l}\text { Target } \\
\text { proteins }\end{array}$ & $\begin{array}{l}\text { Target } \\
\text { phospho }\end{array}$ & sites \\
\hline & 4,138 & 33 & 1,853 & 2,284 & \\
\hline Subnetwork & Motif & $\begin{array}{l}\text { Known } \\
\text { kinase }\end{array}$ & $\begin{array}{l}\text { motifeR } \\
\text { Score }\end{array}$ & $p$-value & $\begin{array}{l}\text { Fold } \\
\text { enrichment }\end{array}$ \\
\hline BIN2 & {$[S / T]-X-X-X-[S / T]$} & GSK3 & 5.53 & $1.96 e-06$ & 1.72 \\
\hline BIN2 & [pS/pT]-P & GSK3/MPK & 317.09 & $1.39 e-30$ & 10.94 \\
\hline MPK4 & {$[S / T]-X-X-X-[S / T]$} & GSK3 & 6.04 & $5.94 \mathrm{e}-07$ & 1.85 \\
\hline MPK4 & [pS/pT]-P & GSK3/MPK & 316.99 & $1.65 e-30$ & 10.90 \\
\hline MPK6 & $P-x-[p S / p T]-P$ & MPK3/6 & 314.00 & $3.56 \mathrm{e}-16$ & 7.70 \\
\hline MPK10 & {$[S / T]-X-X-X-[S / T]$} & GSK3 & 6.33 & $3.15 e-07$ & 2.00 \\
\hline MPK10 & [pS/pT]-P & GSK3/MPK & 311.33 & $3.10 e-14$ & 7.42 \\
\hline
\end{tabular}

Fig. 4. Kinase signaling network. a, A signaling network was inferred using phosphoproteomic data from bin2D, bin2T, and raptor1b mutants. Activated kinases are shown as named circles with their size representing the number of predicted targets (i.e., node outdegree). Target proteins are represented as small, purple circles. b, De novo motif enrichment analysis among predicted direct targets (i.e., node first neighbors) for BIN2, MPK4, and MPK10 showed high enrichment for the GSK3 motif ([S/T]-X-X-X-[S/T]) and GSK3/MPK3 motif ([pS/pT]-P). Analysis on MPK6 predicted direct targets showed significant enrichment of MPK3/6 motif (P-X-[pS/pT]P). Enrichment analysis was done on a 14 amino acids window, centered on target phoshposites. 
ported in the literature and present in our network were correctly predicted as BIN2 targets. These results support the target prediction value of our inferred kinase signaling network.

\section{Phosphoproteomics and kinase-signaling network reconstruction reveal proteins required for normal BR response and autophagy. After analyzing the phospho-} proteome of our bin2 and raptor $1 b$ mutants and predicting their signaling network, the next logical step was to determine whether these predictions identified proteins bridging both BR response and TORC-related autophagy. To assess this question, we enlisted those proteins being differentially phosphorylated simultaneously in either of the BIN2 mutants (i.e., bin2D or bin2T) and in raptor1b (Supplementary Fig. 6a,b). When selecting candidates, we focused our attention first on those proteins upphosphorylated in bin2D and those down-phosphorylated in bin2T since this phosphorylation "directionality" could pinpoint those proteins direct or indirectly affected by BIN2 activity. We then fine-tuned this selection by keeping only those proteins exhibiting differential phosphorylation in raptor1b (Supplementary Fig. 6a,b). From this universe, we selected 42 candidate genes present in our kinase-signaling network (either as a target, an activated kinase, or both) and obtained mutants to assess their BR and autophagy phenotypes (Supplementary Data Set 11). Mutant lines for these candidate genes were tested for hypocotyl elongation in response to $B L$ as a means to assess their sensitivity to BR. From the tested candidate genes, $33.4 \%$ (14/42) showed a significantly altered BL response (Fig. 5a and Supplementary Data Set 11a). To assess autophagy levels as a readout of TORC activity, a total of 28 candidate genes that showed significantly altered hypocotyl elongation in response to $\mathrm{BL}$, exhibited decreased phosphorylation in raptor $1 b$ mutant, or both, were stained using the fluorescent dye monodansylcadaverine (MDC) (Floyd et al., 2015). The number of acidic vesicles as a proxy for the number of autophagosomes occurring in the root elongation zone was then assessed by microscopy (Floyd et al., 2015). Twenty genes $(71.4 \%$ of assayed candidate genes) showed consistently increased autophagy levels when mutated (Fig. 5a and Supplementary Data Set 11b). We further confirmed the autophagy phenotype on selected mutants by examining GFP-ATG8e marker, which labels autophagosomal membranes, by transient expression in protoplasts obtained from mutant lines or in stable GFPATG8e transgenic lines in the mutant backgrounds (Xiong et al., 2007; Floyd et al., 2015). From this confirmation process, $100 \%$ of assayed mutants showed increased autophagy when compared to WT (Supplementary Data Set 11c). Additionally, mutants for the six genes without significant increase in autophagy under normal conditions were subjected to sucrose starvation and stained with MDC. All six mutants showed a reduced induction of autophagy upon starvation when compared to WT, with four showing a complete loss of induction (Fig. 5a and Supplementary Data Set 11d).

In summary, we found a total of 26 genes out of the 42 selected candidates $(61.9 \%)$ with a significant response to BR and/or altered level of autophagy (Supplementary Table 1). These results confirm the robustness of our pipeline as a way of selecting candidate proteins related to the brassinosteroid and/or autophagy pathways and, possibly, participating in plant growth/stress balance orchestrated by BIN2 and TORC.

\section{Discussion}

Brassinosteroid and TORC have emerged as two key signaling pathways coordinating growth and stress responses. To gain a global view of $B R$ and TORC crosstalk, we performed deep multi-omics profiling of the transcriptome, proteome, and phosphoproteome of bin2 and raptor1b mutants. Our results provided evidence that BR and TORC pathways crosstalk at system-wide levels as they affect a common set of genes. Using the multi-omics data, we generated a kinase-signaling network, which was used to predict important regulators functioning in BR/TORC pathways. Our genetic studies indeed confirmed that many of the genes predicted from the network play important roles in BR-regulated growth and/or autophagy.

Our work supports previous transcriptome profiling studies of BR including (Wang et al., 2014; Kim et al., 2019; Liu et al., 2020) and TOR signaling (Ren et al., 2012; Xiong et al., 2013; Dong et al., 2015). Despite long-standing interest in BRs, comprehensive (phospho)proteomic profiles examining BR signaling are limited and proteome-wide identification of substrates of the key regulatory kinase BIN2 is lacking. By profiling bin2 mutants, we identified transcripts, proteins, and phosphorylation sites whose proper expression is dependent on BIN2. Furthermore, using MAKS we provide a global catalogue of potential direct BIN2 substrates. In terms of TORC signaling, we substantially expand on the work of Salem et al., which provided an initial description of proteins that are mis-expressed in raptor1b (Salem et al., 2018) as well as the proteins and phosphorylation sites that respond to TOR inhibition via treatment with Torin 2, AZD8055, or rapamycin (Van Leene et al., 2019; Scarpin et al., 2020). Among our raptor1b 2,338 DE phosphosites, we found $46.8 \%$ (37/79) of the TOR-regulated phosphoproteins and seven of the TOR-interacting proteins from Van Leene et al, and $60.0 \%(48 / 80)$ of the Torin2 responsive phosphoproteins reported by Scarpin et al. Most importantly, through the generation and analysis of these multi-omics data we found a significant overlap of gene-products (i.e., transcript, protein, or phosphosites) that are regulated by both BIN2 and RAPTOR1B. Together our data suggest that a shared regulatory core exists between $B R$ and TORC signaling. 
Using our phosphoproteomics data, we inferred kinases that are differentially active in the analyzed mutant backgrounds. By assessing correlation between kinase activation and substrate phosphorylation levels, we reconstructed a kinase-signaling network predicting the regulatory linkages. Focusing on targets predicted by this regulatory network, and accounting for the phosphosites fold-change "directionality" on each mutant, we identified and tested a set of candidate genes for their involvement in BR/TORC signaling pathways. Mutants for forty-two genes were assayed and 26 of them showed an altered phenotype (Supplementary Table 1). To summarize these phenotyping results, we divided our gene set into 5 groups according to their different phenotypes in BR-regulated hypocotyl elongation and autophagy levels as a readout of TORC activity (Fig. 5b).

TOR signaling is known to be positively regulated by auxin and glucose availability (Xiong et al., 2013; Li et al., 2017; Schepetilnikov et al., 2017). Here, we found the auxin efflux carrier PIN4 and the chloroplast protein importer TOC159 as part of group I (autophagy negative regulators, Fig. 5b). This finding agrees with their corresponding functions: PIN4 creates an auxin sink at the cells below the quiescent center, a crucial event for normal auxin distribution (Friml et al., 2002). Additionally, TOC159 is essential for chloroplast biogenesis and, therefore, photosynthetic activity (Bauer et al., 2000). In parallel with this, we discovered homologs of proteins involved in autophagy and TOR signaling in human. Homo sapiens (Hsa) PTEN has been shown to negatively regulate both mTOR signaling and autophagy through independent pathways (Errafiy et al., 2013). Similarly, our results show Arabidopsis PTEN3 as an autophagy negative regulator (group I, Fig. 5). On the other hand, HsaHMGB1 can translocate to the cytoplasm and induce autophagy upon perception of reactive oxygen species (Tang et al., 2010). Here, HMGB1 is part of group II (positive BR regulator and negative autophagy regulation), suggesting an opposite function in plants.

Finally, our results identified previously unknown genes involved in BR and autophagy pathways. For example, mutant phenotype analysis indicated that MPK6 functions as a positive BR regulator and negative autophagy regulator. Although no direct role as a BR-induced growth has been established, MPK6 kinase is involved in a myriad of processes and has been shown to directly phosphorylate and activate BES1 to increase immune response (Kang et al., 2015). Furthermore, BIN2 can phosphorylate and inhibit MKK4, a direct MPK6 activator (Khan et al., 2013). Moreover, our signaling network prediction situates MPK6 as a possible RAPTOR1B regulator. Many other genes play a role in both BR-regulated growth and modulation of autophagy were identified by our analysis (Fig. 5b).
In summary, this study builds upon previous findings that BR and TORC crosstalk in the regulation of plant growth and stress responses (Zhang et al., 2014, 2016; Nolan et al., 2017b; Xiong et al., 2017; Salem et al., 2018; Vleesschauwer et al., 2018; Liao and Bassham, 2020; Nolan et al., 2020). Our multi-omics studies provide genome-wide evidence for extensive interactions between BR and TORC signaling pathways across three different regulatory layers (i.e., transcript, protein, and phosphorylation). We inferred a kinase signaling network that can effectively predict genes involved in these two pathways. Further functional studies should help refine the predicted regulatory network and define the mechanisms by which these regulators modulate both BR and TORC-regulated processes.

\section{Methods}

Plant material. Arabidopsis thaliana mutant lines bin2-1 (Li and Nam, 2002), bin2-3 bil1 bil2 (Yan et al., 2009), and raptor1-1 (Anderson et al., 2005; Deprost et al., 2005) were used in this research as bin2D, bin2 $T$, and raptor $1 b$, respectively. The full list of seed stocks used in this work are summarized in Supplementary Data Set 12. All plants were grown in LC1 soil mix (Sungro) under long day conditions $\left(16 \mathrm{~h}\right.$ light, $22^{\circ} \mathrm{C}$ ) unless said otherwise. Columbia- 0 ecotype was used as wild-type control for all assays.

QuantSeq library preparation and sequencing. Four biological replicates of 20-day-old rosette leaves were collected from WT and each mutant (bin2T, bin2D, and raptor1b) and immediately frozen in liquid N2. Tissue was ground for at least 15 minutes under liquid N2 using mortar and pestle. Total RNA was extracted using RNAeasy Plant Mini Kit with DNasel treatment (Qiagen). Five-hundred ng of total RNA was used for QuantSeq 3' (Moll et al., 2014) mRNA-seq library construction kit (Lexogen). Library sequencing was performed on an Illumina HiSeq3000. An average of 2,047,038 100-bp, single end reads was obtained per sample.

Transcriptomic data analysis. Data processing followed the pipeline suggested by QuantSeq manufacturer. Briefly, reads were adapter- and quality-trimmed using BBDuk v37.36. Trimmed reads were mapped to the Arabidopsis reference transcriptome (TAIR10 annotation) using Star Aligner v2.5.3a (Dobin et al., 2013). Finally, transcript counts were extracted using HTSeq-count v0.11.2 (Anders et al., 2015). Differential expression was assessed using the PoissonSeq R package ( $\mathrm{Li}$ et al., 2012). A q-value < 0.05 and absolute fold change $>1.32$ was used as cutoff for designating differentially expressed transcripts. All data processing scripts were deposited in a github repository (see Data Availability section)

Protein extraction for global proteome and phosphoproteome profiling. Three biological replicates from the same tissue collected for transcriptome analysis were used for protein extraction, using the urea-FASP method 


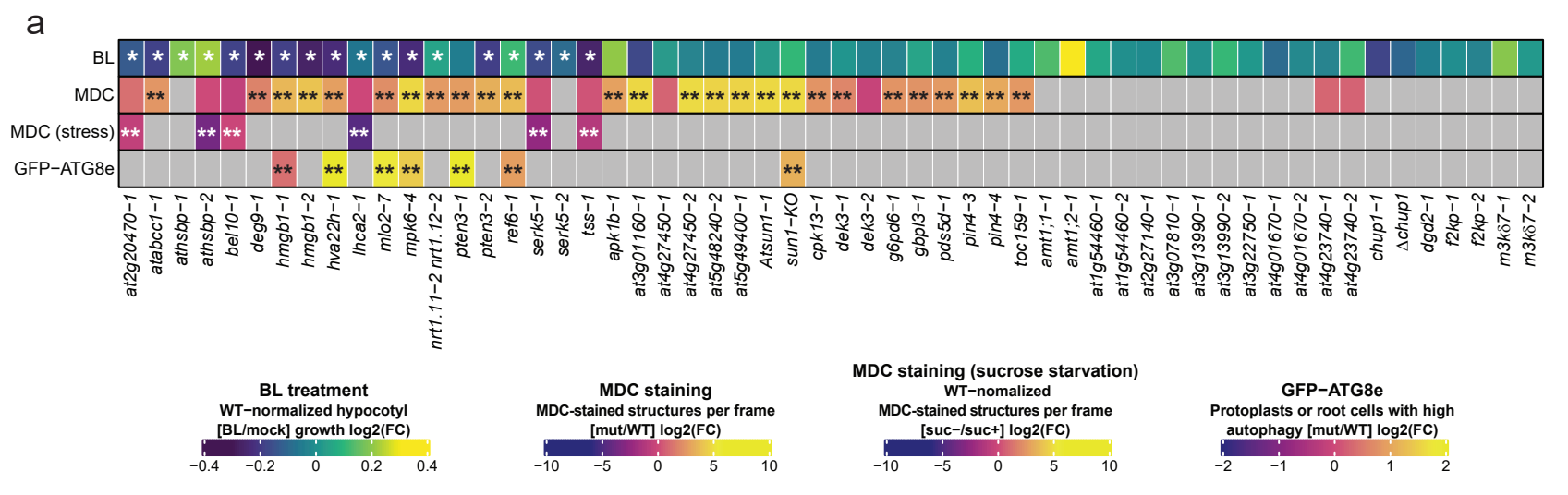

b

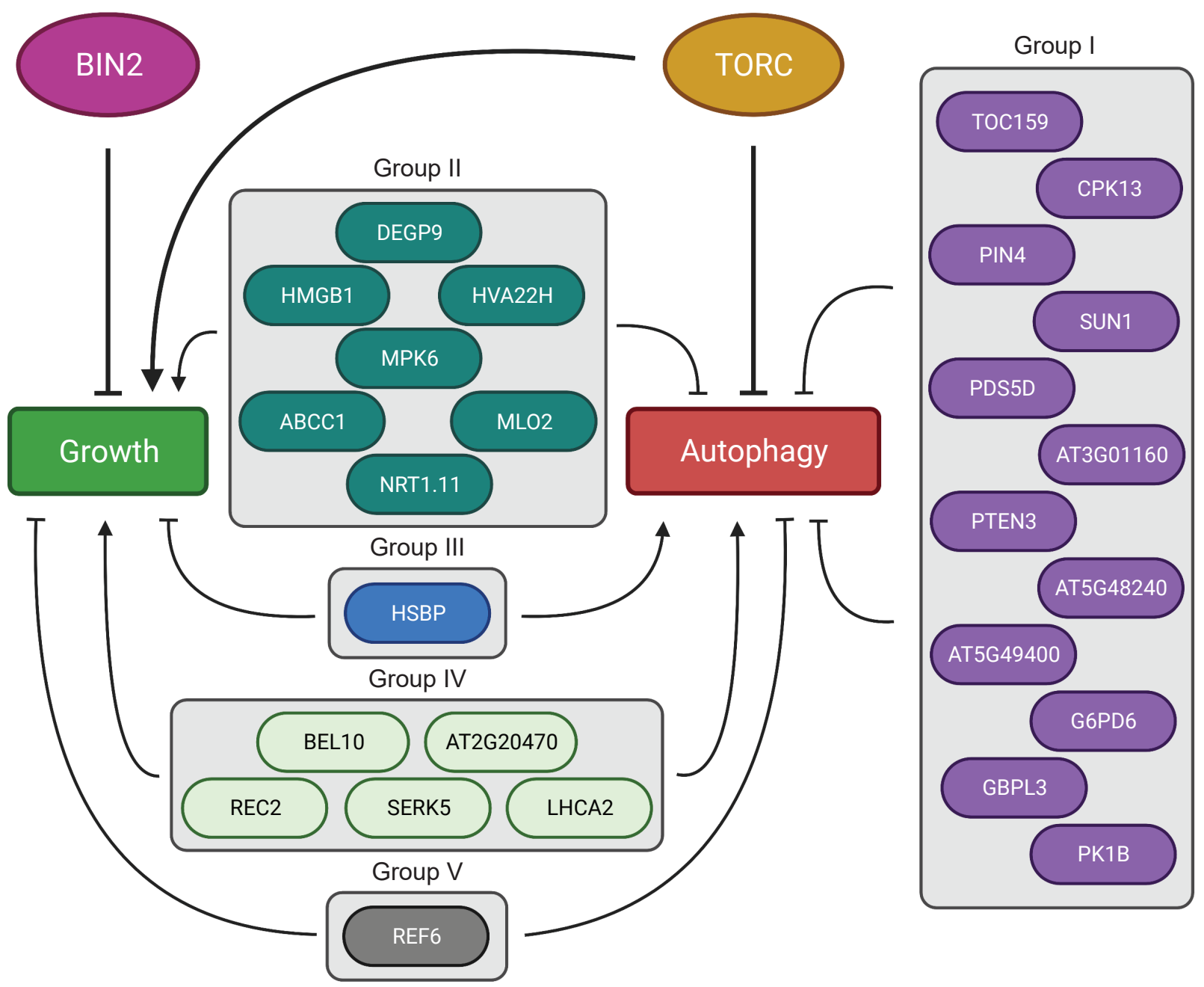

Fig. 5. Identification of candidate genes with altered BR response and autophagy. a, (first row) Hypocotyl length response to BL treatment in selected mutant lines (four biological replicates each with $n=6$ ). Values shown are the log2 fold change in hypocotyl length (BL/Mock). * $p<0.1$, generalized linear model. (second and third row) Number of MDC-stained structures in selected mutants in normal growth conditions (second row, $n=10$ ) or sucrose starvation (third row, $n=10$ ). Values shown are the log2 fold change in the number of MDC-stained structures (mutant/WT, second row; -suc/+suc, third row). ${ }^{* *} p<0.05$, two-sample t-test (fourth row) GFP-ATG8e expression in selected mutants. Values shown are the log2 fold change in the number of cells expressing GFP-ATG8e (mutant/WT). ${ }^{* *} p<0.05$, two-sample t-test. $\mathbf{b}$, Genes with significant response to BL or altered autophagy levels are organized into five groups according to their mutants' phenotype. 
described by (Song et al., 2018a, 2018b, 2020) as follows: $250 \mathrm{mg}$ of finely ground tissue was subjected to mechanical disruption using $250 \mathrm{mg} \mathrm{ZrO}_{2}$ beads in presence of $500 \mu \mathrm{L}$ lysis buffer (8M urea; $100 \mathrm{mM}$ TRIS-HCl, $\mathrm{pH}$ 7.0; $5 \mathrm{mM}$ TCEP) on a MiniG tissue homogenizer (Spex SamplePrep). Sample was clarified, and supernatant was transferred to a clean tube. Proteins were precipitated using rounds of 45 -minute incubation at $-80^{\circ} \mathrm{C}$ as follows: 1 round of ice-cold $100 \%$ acetone, 2 rounds of $80 \%$ acetone and 3 rounds of $0.2 \mathrm{mM} \mathrm{Na}_{3} \mathrm{VO}_{4}$ in $100 \%$ methanol. Each round of incubation consisted of sample resuspension assisted by probe sonication, incubation at $-20^{\circ} \mathrm{C}$, centrifugation at 4,500xG for 10 minutes, and removal of supernatant. Extracted proteins were resuspended by sonication in urea resuspension buffer (URB, 8M urea in $50 \mathrm{mM}$ TRIS-HCl, $\mathrm{pH}=7.0 ; 5 \mathrm{mM}$ TCEP; $1 x$ phosphatase inhibitor cocktail) and further cleaned through Filter-Assisted Sample Preparation (FASP) using Amicon Ultra-4 30kDa MWCO filter units (Millipore) in the presence of UA buffer (8M urea in 100mM TRIS- $\mathrm{HCl}, \mathrm{pH}=8.0 ; 1 \times$ phosphatase inhibitor cocktail). Samples were reduced with $2 \mathrm{mM}$ TCEP, alkylated using $50 \mathrm{mM}$ iodoacetamide (IAM) and digested into peptides using one round of overnight incubation at $37^{\circ} \mathrm{C}$ with 1:50 (enzyme:protein) trypsin (Roche, Cat. No. 03708969001) and a second round of incubation for 4 hours at $37^{\circ} \mathrm{C}$ with trypsin and Lys-C (Wako Chemicals, Catalog number 125-05061). Purified samples were further desalted using SepPack C18 columns (Waters). Tandem Mass Tag (TMT, Thermo Scientific) labeling was performed on $330 \mu \mathrm{g}$ of purified peptides from each sample as previously reported (Song et al., 2020). TMT labeling reaction was stopped using $5 \%$ hydroxylamine and the quenched samples were then pooled. One hundred $\mu \mathrm{g}$ of labeled peptide was set aside for global proteome profiling and the remaining labeled sample was subjected to a second round of $\mathrm{C} 18$ desalting before phosphopeptide enrichment. Serial Metal Oxide Affinity Chromatography (SMOAC) method from Thermo Scientific was used for phosphopeptide enrichment. Briefly, High-Select $\mathrm{TiO}_{2}$ Phosphopeptide Enrichment kit (Thermo Scientific) was used as a first enrichment step and all flow-through was pooled, concentrated to almost dry on a SpeedVac and used as input for High Select Fe-NTA Phosphoptide Enrichment kit (Thermo Scientific). Phosphopeptides obtained from both enrichment processes were dried using a SpeedVac, resuspended in $0.1 \%$ formic acid in Optima grade $\mathrm{H}_{2} \mathrm{O}$ (Millipore) and pooled together.

BIN2 Multiplexed Assay for Kinase Specificity. The MAKS as was performed based on the protocol described by (Jayaraman et al., 2017). Protein was extracted for MAKS from $1 \mathrm{~g}$ of leaf ground tissue from 20-days-old wild type Arabidopsis plants using the phenol-FASP protocol as described previously (Song et al., 2018b, 2020). Three $\mathrm{mg}$ of total purified protein was resuspended in URB, re-precipitated in ice-cold $100 \mathrm{mM} \mathrm{NH}_{4} \mathrm{CH}_{3} \mathrm{CO}_{2}$ in $100 \%$ methanol, and resuspended in kinase buffer $(50 \mathrm{mM}$
TRIS- $\mathrm{HCl}, \mathrm{pH}=7.7 ; 5 \mathrm{mM} \mathrm{MgCl} 2 ; 5 \mathrm{mM}$ ATP; $1 \mathrm{x}$ phosphatase inhibitor cocktail). Resuspended protein was divided into $600 \mu \mathrm{g}$ aliquots and incubated with either recombinant GST or GST-BIN2 at a 1:75 (enzyme:protein) ratio at $37^{\circ} \mathrm{C}$ with gentle shaking for 1 hour. After incubation, protein solution was subjected to FASP, reduced with $2 \mathrm{mM}$ TCEP, alkylated in 50mM IAM, and digested using trypsin as described by (Song et al., 2020). Three replicates were made for each treatment (i.e., GST and GST-BIN2). Two hundred $\mu \mathrm{g}$ of peptides from each replicate were used for TMT labeling. Phosphopeptide enrichment was performed on labeled peptides using SMOAC as described previously in this paper. Cloning of GST-BIN2 was described in (Yin et al., 2002); The fusion protein was purified using Glutathione agarose beads as described in (Jiang et al., 2019).

LC-MS/MS. Chromatography was performed on an Agilent 1260 quaternary HPLC with constant flow rate of $600 \mathrm{~nL}$ $\mathrm{min}^{-1}$ achieved via a splitter. A Sutter P-2000 laser puller was used to generate sharp nanospray tips from $200 \mu \mathrm{m}$ ID fused silica capillary. Columns were all in-house packed on a Next Advance pressure cell using $200 \mu \mathrm{m}$ ID capillary. All samples were loaded into a $10 \mathrm{~cm}$ capillary column packed with $5 \mu \mathrm{M}$ Zorbax SB-C18 (Agilent) and then connected to a $5 \mathrm{~cm}$-long strong cationic exchange (SCX) column packed with $5 \mu \mathrm{M}$ PolySulfoethyl. The SCX column was then connected to a $20 \mathrm{~cm}$ long nanospray tip, packed with $2.5 \mu \mathrm{m} \mathrm{C18}$ (Waters). For global protein abundance, $45 \mu \mathrm{g}$ of labeled peptides and 24 ammonium acetate salt steps of 150 min each were used. For phosphoproteomics, $25 \mu \mathrm{g}$ of enriched peptides and 15 salt steps were used. For MAKS, $30 \mu \mathrm{g}$ of enriched peptides and 18 salt steps were used. Spectra were obtained using a Thermo Scientific Q-Exactive Plus high-resolution quadrupole-Orbitrap mass spectrometer on data-dependent mode as previously described (Zhang et al., 2019).

Proteomics data analysis. Spectra for global protein abundance runs were searched using the Andromeda Search Engine (Cox et al., 2011) against the TAIR10 Arabidopsis proteome (https://www arabidopsis. org/download_files/Proteins/TAIR10_ protein_lists/TAIR10_pep_20101214) on MaxQuant software v1.6.1.0 (Tyanova et al., 2016) as reported previously (Zhang et al., 2019). Sample loading and internal reference scaling normalization methods were used to account for differences within and between LC-MS/MS runs, respectively (Plubell et al., 2017). Differential expression was assessed using the PoissonSeq $\mathrm{R}$ package ( $\mathrm{Li}$ et al., 2012). A q-value $<0.1$ was used as cutoff for designating differentially expressed proteins. Scripts for data analysis were deposited in a github repository (see Data Availability section).

Phosphoproteomics data analysis. Spectra for both bin2/raptor1b mutant profling and MAKS were searched together using similar approach as with global protein 
abundance with exceptions. Briefly, MaxQuant software v1.6.10.43 was used instead and "Phospho (STY)" search for variable modifications was included. Sample loading and internal reference scaling normalization methods were used to account for differences within and between LCMS/MS runs, respectively (Plubell et al., 2017). Differential expression was assessed using the edgeR $R$ package (Robinson et al., 2010). A q-value $<0.1$ was used as cutoff for designating differential phosphorylation. See Data Availability section for the full analysis script.

Motif enrichment analysis. Motif enrichment was performed using the motifeR R package (Wang et al., 2019) with default settings, serine or threonine as the central residues, a $p$-value threshold of 0.001 , and TAIR 10 protein annotation as background reference. Enrichment $p$-value was calculated using hypergeometric testing.

Analysis of overlap between BIN2 MAKS and bin2 mutant datasets. To find overlapping phosphosites, we defined any two distinct phosphosites as identical if they originated from the same phosphoprotein and were less than 10 amino acid residues apart. This approach was used to account for cases where phosphosites were not localized to a specific amino acid on a given peptide. Overlap statistical significance was assessed by hypergeometric test.

\begin{abstract}
Kinase activation loop prediction. Protein kinases were identified using a modified version of the pipeline described by (Walley et al., 2013). Briefly, all 35,386 protein sequences available in the TAIR10 annotation (https://www.arabidopsis.org/download_ files/Proteins/TAIR10_protein_lists/ TAIR10_pep_20101214) were searched for kinase domain using The National Center for Biotechnology Information batch conserved domain search tool (Lu et al., 2020). From this list of 1,522 proteins with identified kinase domain, 878 were also annotated with activation loop (A-loop) coordinates by the search tool. The kinase domains of proteins lacking the A-loop coordinates were aligned using MAFFT (Katoh and Standley, 2013) and the well conserved A-loop beginning (DFG) and end (APE) motifs were manually searched. An extra 482 A-loop coordinates were obtained, for a total of 1,360 protein kinases with A-loop coordinates.
\end{abstract}

Kinase-substrate network. Kinases with differential phosphorylation inside the A-loop (activated kinases) were used as regulators to build the kinase-substrate network. For this, the Spearman and Pearson correlation between a regulator and the rest of differentially phosphorylated peptides was calculated as described previously (Walley et al., 2013).

BL response assays. Seeds were vapor-phase sterilized in chlorine gas, stratified at $4^{\circ} \mathrm{C}$ for 1 week, and germinated in half-strength Linsmaier and Skoog media (Cais- son Labs, catalog number LSP03) supplemented with either DMSO or $100 \mathrm{nM}$ brassinolide (BL). Seedlings were grown for 7 days at $22 / 18^{\circ} \mathrm{C}$ (day/night), 16 hours of light, $40 \%$ relative humidity, and light intensity of $120 \mu \mathrm{mol} \mathrm{m} \mathrm{m}^{-2}$ $\mathrm{s}^{-1}$. Seedlings were imaged and hypocotyl length was measured using Fiji software (Schindelin et al., 2012). A generalized linear model with treatment and genotype as factors and controlling for random effects of replicate and plate was applied and a threshold of "genotype by treatment interaction" $p$-value $<0.1$ was set as significance cutoff. Four replicates were made with six seedlings being measured for each replicate. The whole experiment was repeated at least two times per genotype.

MDC staining. Seven-day-old Arabidopsis seedling roots were stained with monodansylcadaverine (MDC, Sigma) as described previously (Contento et al., 2005). Cells within the root elongation zone for ten seedlings were observed and imaged using a Zeiss Axio Imager.A2 upright microscope (Zeiss) equipped with Zeiss Axiocam $\mathrm{BW} /$ color digital cameras and a DAPI-specific filter at the lowa State University Microscopy and Nanoimaging Facility. The number of MDC-labeled puncta in each image was counted and averaged from at least 10 images per sample. For sucrose starvation, 7-day-old seedlings grown on solid $1 / 2$ MS medium were transferred to solid $1 / 2$ MS medium lacking sucrose and kept in darkness for an additional 3 days before staining. Significance was assessed through two-sample t-test for MDC staining under normal growing conditions, whereas a generalized linear model with treatment and genotype as factors and controlling for random effects of replicates was used for MDC staining under sucrose starvation. A $p$-value $<0.05$ was used a cutoff on both cases.

GFP-ATG8e protoplast assay and stable lines. Protoplasts were extracted from leaves from 20-day-old plants and transformed as described previously (Wu et al., 2009). Protoplasts were observed on a LSM-700 confocal microscope (Zeiss) using a FITC filter, and protoplasts with more than three visible autophagosomes were counted as active for autophagy as previously described (Yang et al., 2016; Pu et al., 2017). One hundred protoplasts were analyzed per genotype and the experiment was repeated three times. Homozygous mutant lines were crossed to plants expressing GFP-ATG8e and stable lines were obtained. Roots from GFP-ATG8e-expressing lines that were also homozygous for the mutation were analyzed on LSM-700 confocal microscope (Zeiss) using a FITC filter. Cells within the root elongation zone of no less than fifteen seedlings were photographed and the number of autophagosomes in each image was counted and averaged from at least 10 images per sample. Significance was assessed in both cases by two-sample t-test and a $p$-value $<$ 0.05 was used as cutoff.

Data Availability. The RNA-seq data generated by this work has been deposited at the National Center for 
bioRxiv preprint doi: https://doi.org/10.1101/2021.02 12 431003; this version posted February 13, 2021. The copyright holder for this preprint (which was not certified by peer review) is the author/funder, who has granted bioRxiv a license to display the preprint in perpetuity. It is made available under aCC-BY-NC-ND 4.0 International license.

Biotechnology Information (NCBI) Short Read Archive (SRA) as BioProject accession number PRJNA678744. The original MS proteomics raw data, as well as the MaxQuant output files, can be downloaded from MassIVE (http://massive.ucsd.edu) using the identifier MSV000086460 for bin2D, bin2T, and raptor1b mutants profiling and MSV000086462 for the BIN2 MAKS. All the scripts used for this work are available on the following github repository: https://github.com/ chrisfmontes/BIN2_RAPTOR1B_MULTIOMICS.

\section{ACKNOWLEDGEMENTS}

This work was supported by the lowa State University Plant Science Institute (YY and JWW), NIH R01GM120316 (YY, DB, JWW), NSF IOS-1818160 (YY and JWW), and USDA NIFA Hatch project IOW3808 funds to JWW. NMC is supported by a USDA NIFA Postdoctoral Research Fellowship (201967012-29712) and TMN is supported by the National Science Foundation Postdoctoral Research Fellowships in Biology Program (Grant No. IOS-2010686).

This manuscript was formatted in Overleaf using the Henriques Lab bioRxiv template. 


\section{References}

Adams, J.A. (2003). Activation loop phosphorylation and catalysis in protein kinases: Is there functional evidence for the autoinhibitor model? Biochemistry 42: 601-607.

Ahiri, A. (2019). Insights into evolutionary interaction patterns of the "Phosphorylation Activation Segment" in kinase. Bioinformation 15: 666-677.

Amanchy, R., Periaswamy, B., Mathivanan, S., Reddy, R., Tattikota, S.G., and Pandey, A. (2007). A curated compendium of phosphorylation motifs. Nature Biotechnology 25: 285-286.

Anders, S., Pyl, P.T., and Huber, W. (2015). HTSeq-a Python framework to work with high-throughput sequencing data. Bioinformatics 31: 166-169.

Anderson, G.H., Veit, B., and Hanson, M.R. (2005). The Arabidopsis AtRaptor genes are essential for post-embryonic plant growth. BMC Biology 3: 12.

Bauer, J., Chen, K., Hiltbunner, A., Wehrli, E., Eugster, M., Schnell, D., and Kessler, F. (2000). The major protein import receptor of plastids is essential for chloroplast biogenesis. Nature 403: 203-207.

Beekhof, R. et al. (2019). INKA, an integrative data analysis pipeline for phosphoproteomic inference of active kinases. Molecular Systems Biology 15: e8250.

Bozhkov, P.V. (2018). Plant autophagy: mechanisms and functions. Journal of Experimental Botany 69: 1281-1285.

Brumbaugh, J., Russell, J.D., Yu, P., Westphall, M.S., Coon, J.J., and Thomson, J.A. (2014). NANOG Is Multiply Phosphorylated and Directly Modified by ERK2 and CDK1 In Vitro. Stem Cell Reports 2: 18-25.

Cai, Z., Liu, J., Wang, H., Yang, C., Chen, Y., Li, Y., Pan, S., Dong, R., Tang, G., Barajas-Lopez, J. de D., Fujii, H., and Wang, X. (2014). GSK3-like kinases positively modulate abscisic acid signaling through phosphorylating subgroup III SnRK2s in Arabidopsis. Proceedings of the National Academy of Sciences of the United States of America 111: 9651-6.

Cho, H. et al. (2014). A secreted peptide acts on BIN2-mediated phosphorylation of ARFs to potentiate auxin response during lateral root development. Nature Cell Biology 16: 66-76.

Clouse, S.D., Langford, M., and McMorris, T.C. (1996). A Brassinosteroid-Insensitive Mutant in Arabidopsis thaliana Exhibits Multiple Defects in Growth and Development. Plant Physiology 111: 671-678.

Contento, A.L., Xiong, Y., and Bassham, D.C. (2005). Visualization of autophagy in Arabidopsis using the fluorescent dye monodansylcadaverine and a GFP-AtATG8e fusion protein. The Plant Journal 42: 598-608.

Cox, J., Neuhauser, N., Michalski, A., Scheltema, R.A., Olsen, J. V., and Mann, M. (2011). Andromeda: A Peptide Search Engine Integrated into the MaxQuant Environment. Journal of Proteome Research 10: 1794-1805.

Deprost, D., Truong, H.-N., Robaglia, C., and Meyer, C. (2005). An Arabidopsis homolog of RAPTOR/KOG1 is essential for early embryo development. Biochemical and Biophysical Research Communications 326: 844-850.

Dobin, A., Davis, C.A., Schlesinger, F., Drenkow, J., Zaleski, C., Jha, S., Batut, P., Chaisson, M., and Gingeras, T.R. (2013). STAR: ultrafast universal RNA-seq aligner. Bioinformatics 29:
15-21.

Dong, P., Xiong, F., Que, Y., Wang, K., Yu, L., Li, Z., and Ren, M. (2015). Expression profiling and functional analysis reveals that TOR is a key player in regulating photosynthesis and phytohormone signaling pathways in Arabidopsis. Frontiers in Plant Science 6: 677.

Errafiy, R., Aguado, C., Ghislat, G., Esteve, J.M., Gil, A., Loutfi, M., and Knecht, E. (2013). PTEN increases autophagy and inhibits the ubiquitin-proteasome pathway in glioma cells independently of its lipid phosphatase activity. PLoS One 8: e83318.

Fiol, C.J., Mahrenholz, A.M., Wang, Y., Roeske, R.W., and Roach, P.J. (1987). Formation of protein kinase recognition sites by covalent modification of the substrate. Molecular mechanism for the synergistic action of casein kinase II and glycogen synthase kinase 3. J. Biol. Chem. 262: 14042-14048.

Floyd, B.E., Morriss, S.C., Maclntosh, G.C., and Bassham, D.C. (2015). Evidence for autophagy-dependent pathways of rRNA turnover in Arabidopsis. Autophagy 11: 2199-2212.

Friml, J., Benková, E., Blilou, I., Wisniewska, J., Hamann, T., Ljung, K., Woody, S., Sandberg, G., Scheres, B., Jürgens, G., and Palme, K. (2002). AtPIN4 Mediates Sink-Driven Auxin Gradients and Root Patterning in Arabidopsis. Cell 108: 661-673.

Fu, L., Wang, P., and Xiong, Y. (2020). Target of Rapamycin Signaling in Plant Stress Responses. Plant Physiology 182: 1613-1623.

Gampala, S.S. et al. (2007). An Essential Role for 14-3-3 Proteins in Brassinosteroid Signal Transduction in Arabidopsis. Developmental Cell 13: 177-189.

Gruszka, D. (2018). Crosstalk of the brassinosteroid signalosome with phytohormonal and stress signaling components maintains a balance between the processes of growth and stress tolerance. International Journal of Molecular Sciences 19: 2675. Guo, H., Li, L., Aluru, M., Aluru, S., and Yin, Y. (2013). Mechanisms and networks for brassinosteroid regulated gene expression. Current Opinion in Plant Biology 16: 545-553.

Hara, K., Maruki, Y., Long, X., Yoshino, K. ichi, Oshiro, N., Hidayat, S., Tokunaga, C., Avruch, J., and Yonezawa, K. (2002). Raptor, a binding partner of target of rapamycin (TOR), mediates TOR action. Cell 110: 177-189.

Hogrebe, A., von Stechow, L., Bekker-Jensen, D.B., Weinert, B.T., Kelstrup, C.D., and Olsen, J.V. (2018). Benchmarking common quantification strategies for large-scale phosphoproteomics. Nature Communications 9: 1045.

Hu, Y. and Yu, D. (2014). BRASSINOSTEROID INSENSITIVE2 interacts with ABSCISIC ACID INSENSITIVE5 to mediate the antagonism of brassinosteroids to abscisic acid during seed germination in Arabidopsis. The Plant cell 26: 4394-408.

Huot, B., Yao, J., Montgomery, B.L., and He, S.Y. (2014). Growth-defense tradeoffs in plants: A balancing act to optimize fitness. Molecular Plant 7: 1267-1287.

Jayaraman, D., Richards, A.L., Westphall, M.S., Coon, J.J., and Ané, J.-M. (2017). Identification of the phosphorylation targets of symbiotic receptor-like kinases using a high-throughput multiplexed assay for kinase specificity. The Plant Journal 90: 1196-1207. 
Jiang, H., Tang, B., Xie, Z., Nolan, T., Ye, H., Song, G., Walley, J., and Yin, Y. (2019). GSK 3-like kinase BIN 2 phosphorylates $\mathrm{RD} 26$ to potentiate drought signaling in Arabidopsis. The Plant Journal 100: 923-937.

Kang, S., Yang, F., Li, L., Chen, H., Chen, S., and Zhang, J. (2015). The Arabidopsis Transcription Factor BRASSINOSTEROID INSENSITIVE1-ETHYL METHANESULFONATESUPPRESSOR1 Is a Direct Substrate of MITOGEN-ACTIVATED PROTEIN KINASE6 and Regulates Immunity. Plant Physiology 167: 1076-1086.

Katoh, K. and Standley, D.M. (2013). MAFFT Multiple Sequence Alignment Software Version 7: Improvements in Performance and Usability. Molecular Biology and Evolution 30: 772-780.

Khan, M., Rozhon, W., Bigeard, J., Pflieger, D., Husar, S., Pitzschke, A., Teige, M., Jonak, C., Hirt, H., and Poppenberger, B. (2013). Brassinosteroid-regulated GSK3/Shaggy-like Kinases Phosphorylate Mitogen-activated Protein (MAP) Kinase Kinases, Which Control Stomata Development in Arabidopsis thaliana. J Biol Chem 288: 7519-7527.

Kim, H., Shim, D., Moon, S., Lee, J., Bae, W., Choi, H., Kim, K., and Ryu, H. (2019). Transcriptional network regulation of the brassinosteroid signaling pathway by the BES1-TPL-HDA19 co-repressor complex. Planta 250: 1371-1377.

Kim, T.-W., Guan, S., Sun, Y., Deng, Z., Tang, W., Shang, J.-X., Sun, Y., Burlingame, A.L., and Wang, Z.-Y. (2009). Brassinosteroid signal transduction from cell-surface receptor kinases to nuclear transcription factors. Nature Cell Biology 11: 1254-1260. Kim, T.-W., Michniewicz, M., Bergmann, D.C., and Wang, Z.-Y. (2012). Brassinosteroid regulates stomatal development by GSK3-mediated inhibition of a MAPK pathway. Nature 482: 419-422.

Li, J., Nagpal, P., Vitart, V., McMorris, T.C., and Chory, J. (1996). A role for brassinosteroids in light-dependent development of Arabidopsis. Science (New York, N.Y.) 272: 398-401.

Li, J. and Nam, K.H. (2002). Regulation of Brassinosteroid Signaling by a GSK3/SHAGGY-Like Kinase. Science 295: 1299-1301.

Li, J., Witten, D.M., Johnstone, I.M., and Tibshirani, R. (2012). Normalization, testing, and false discovery rate estimation for RNA-sequencing data. Biostatistics 13: 523-538.

Li, J., Zhou, H., Zhang, Y., Li, Z., Yang, Y., and Guo, Y. (2020a). The GSK3-like Kinase BIN2 Is a Molecular Switch between the Salt Stress Response and Growth Recovery in Arabidopsis thaliana. Developmental Cell 55: 367-380.e6.

Li, T., Lei, W., He, R., Tang, X., Han, J., Zou, L., Yin, Y., Lin, H., and Zhang, D. (2020b). Brassinosteroids regulate root meristem development by mediating BIN2-UPB1 module in Arabidopsis. PLOS Genetics 16: e1008883.

Li, X., Cai, W., Liu, Y., Li, H., Fu, L., Liu, Z., Xu, L., Liu, H., Xu, T., and Xiong, Y. (2017). Differential TOR activation and cell proliferation in Arabidopsis root and shoot apexes. PNAS 114: 2765-2770.

Liao, C.-Y. and Bassham, D.C. (2020). Combating stress: the interplay between hormone signaling and autophagy in plants. Journal of Experimental Botany 71: 1723-1733.

Lin, L.-L., Hsu, C.-L., Hu, C.-W., Ko, S.-Y., Hsieh, H.-L.,
Huang, H.-C., and Juan, H.-F. (2015). Integrating Phosphoproteomics and Bioinformatics to Study Brassinosteroid-Regulated Phosphorylation Dynamics in Arabidopsis. BMC genomics 16: 533.

Liu, X., Yang, H., Wang, Y., Zhu, Z., Zhang, W., and Li, J. (2020). Comparative transcriptomic analysis to identify brassinosteroid response genes. Plant Physiology 184: pp.00386.2020.

Lu, S. et al. (2020). CDD/SPARCLE: the conserved domain database in 2020. Nucleic Acids Research 48: D265-D268.

Marshall, R.S. and Vierstra, R.D. (2018). Autophagy: The Master of Bulk and Selective Recycling. Annual Review of Plant Biology 69: 173-208.

McAlister, G.C., Huttlin, E.L., Haas, W., Ting, L., Jedrychowski, M.P., Rogers, J.C., Kuhn, K., Pike, I., Grothe, R.A., Blethrow, J.D., and Gygi, S.P. (2012). Increasing the multiplexing capacity of TMTs using reporter ion isotopologues with isobaric masses. Anal. Chem. 84: 7469-7478.

Moll, P., Ante, M., Seitz, A., and Reda, T. (2014). QuantSeq 3 mRNA sequencing for RNA quantification. Nature Methods 11: i-iii.

Noda, T. and Ohsumi, Y. (1998). Tor, a Phosphatidylinositol Kinase Homologue, Controls Autophagy in Yeast ${ }^{*}$. Journal of Biological Chemistry 273: 3963-3966.

Nolan, T., Chen, J., and Yin, Y. (2017a). Cross-talk of Brassinosteroid signaling in controlling growth and stress responses. The Biochemical journal 474: 2641-2661.

Nolan, T.M., Brennan, B., Yang, M., Chen, J., Zhang, M., Li, Z., Wang, X., Bassham, D.C., Walley, J., and Yin, Y. (2017b). Selective Autophagy of BES1 Mediated by DSK2 Balances Plant Growth and Survival. Developmental Cell 41: 33-46.e7.

Nolan, T.M., Vukašinović, N., Liu, D., Russinova, E., and Yin, Y. (2020). Brassinosteroids: Multidimensional Regulators of Plant Growth, Development, and Stress Responses. The Plant Cell 32: 295-318.

Plubell, D.L., Wilmarth, P.A., Zhao, Y., Fenton, A.M., Minnier, J., Reddy, A.P., Klimek, J., Yang, X., David, L.L., and Pamir, N. (2017). Extended Multiplexing of Tandem Mass Tags (TMT) Labeling Reveals Age and High Fat Diet Specific Proteome Changes in Mouse Epididymal Adipose Tissue. Molecular cellular proteomics: MCP 16: 873-890.

$\mathrm{Pu}$, Y., Luo, X., and Bassham, D.C. (2017). TOR-Dependent and -Independent Pathways Regulate Autophagy in Arabidopsis thaliana. Frontiers in Plant Science 8: 1204. Rayapuram, N. et al. (2021). Chromatin phosphoproteomics unravels a function for AT-hook motif nuclear localized protein AHL13 in PAMP-triggered immunity. PNAS 118.

Ren, M. et al. (2012). Target of Rapamycin Signaling Regulates Metabolism, Growth, and Life Span in Arabidopsis. The Plant Cell 24: 4850-4874.

Robinson, M.D., McCarthy, D.J., and Smyth, G.K. (2010). edgeR: a Bioconductor package for differential expression analysis of digital gene expression data. Bioinformatics 26 : 139-140.

Ryu, H., Kim, K., Cho, H., and Hwang, I. (2010). Predominant actions of cytosolic BSU1 and nuclear BIN2 regulate subcellular localization of BES1 in brassinosteroid signaling. Molecules and 

available under aCC-BY-NC-ND 4.0 International license.

Cells 29: 291-296.

Ryu, H., Kim, K., Cho, H., Park, J., Choe, S., and Hwang, I. (2007). Nucleocytoplasmic shuttling of BZR1 mediated by phosphorylation is essential in Arabidopsis brassinosteroid signaling. Plant Cell 19: 2749-2762. Salem, M.A., Li, Y., Bajdzienko, K., Fisahn, J., Watanabe, M., Hoefgen, R., Schöttler, M.A., and Giavalisco, P. (2018). RAPTOR Controls Developmental Growth Transitions by Altering the Hormonal and Metabolic Balance. Plant physiology 177: 565-593.

Scarpin, M.R., Leiboff, S., and Brunkard, J.O. (2020). Parallel global profiling of plant TOR dynamics reveals a conserved role for LARP1 in translation. eLife $\mathbf{9 .}$

Schepetilnikov, M., Makarian, J., Srour, O., Geldreich, A., Yang, Z., Chicher, J., Hammann, P., and Ryabova, L.A. (2017). GTPase ROP2 binds and promotes activation of target of rapamycin, TOR, in response to auxin. The EMBO Journal 36: 886-903.

Schindelin, J. et al. (2012). Fiji: an open-source platform for biological-image analysis. Nature Methods 9: 676-682.

Schmidlin, T., Debets, D.O., Gelder, C.A.G.H. van, Stecker, K.E., Rontogianni, S., Eshof, B.L. van den, Kemper, K., Lips, E.H., Biggelaar, M. van den, Peeper, D.S., Heck, A.J.R., and Altelaar, M. (2019). High-Throughput Assessment of Kinome-wide Activation States. cels 9: 366-374.e5.

Song, G., Brachova, L., Nikolau, B.J., Jones, A.M., and Walley, J.W. (2018a). Heterotrimeric G-Protein-Dependent Proteome and Phosphoproteome in Unstimulated Arabidopsis Roots. PROTEOMICS 18: 1800323. Song, G., Hsu, P.Y., and Walley, J.W. (2018b). Assessment and Refinement of Sample Preparation Methods for Deep and Quantitative Plant Proteome Profiling. PROTEOMICS 18: 1800220. Song, G., Montes, C., and Walley, J.W. (2020). Quantitative Profiling of Protein Abundance and Phosphorylation State in Plant Tissues Using Tandem Mass Tags. In Plant Proteomics: Methods and Protocols, J.V. Jorrin-Novo, L. Valledor, M.A. Castillejo, and M.-D. Rey, eds, Methods in Molecular Biology. (Springer US: New York, NY), pp. 147-156.

Sreeramulu, S., Mostizky, Y., Sunitha, S., Shani, E., Nahum, H., Salomon, D., Hayun, L.B., Gruetter, C., Rauh, D., Ori, N., and Sessa, G. (2013). BSKs are partially redundant positive regulators of brassinosteroid signaling in Arabidopsis. The Plant Journal 74: 905-919.

Tang, D., Kang, R., Livesey, K.M., Cheh, C.-W., Farkas, A., Loughran, P., Hoppe, G., Bianchi, M.E., Tracey, K.J., Zeh, H.J., and Lotze, M.T. (2010). Endogenous HMGB1 regulates autophagy. Journal of Cell Biology 190: 881-892.

Tyanova, S., Temu, T., and Cox, J. (2016). The MaxQuant computational platform for mass spectrometry-based shotgun proteomics. Nature Protocols 11: 2301-2319.

Van Leene, J. et al. (2019). Capturing the phosphorylation and protein interaction landscape of the plant TOR kinase. Nature Plants 5: 316-327.

Verma, V., Ravindran, P., and Kumar, P.P. (2016). Plant hormone-mediated regulation of stress responses. BMC Plant Biology 16: 1-10.

Vleesschauwer, D.D., Filipe, O., Hoffman, G., Seifi, H.S., Haeck, A., Canlas, P., Bockhaven, J.V., Waele, E.D., De- meestere, K., Ronald, P., and Hofte, M. (2018). Target of rapamycin signaling orchestrates growth-defense trade-offs in plants. New Phytologist 217: 305-319.

Walley, J.W., Shen, Z., Sartor, R., Wu, K.J., Osborn, J., Smith, L.G., and Briggs, S.P. (2013). Reconstruction of protein networks from an atlas of maize seed proteotypes. Proceedings of the National Academy of Sciences 110: E4808-E4817.

Wang, S., Cai, Y., Cheng, J., Li, W., Liu, Y., and Yang, H. (2019). motifeR: An Integrated Web Software for Identification and Visualization of Protein Post-Translational Modification Motifs. PROTEOMICS: 1900245.

Wang, X., Chen, J., Xie, Z., Liu, S., Nolan, T., Ye, H., Zhang, M., Guo, H., Schnable, P.S., Li, Z., and Yin, Y. (2014). Histone Lysine Methyltransferase SDG8 Is Involved in BrassinosteroidRegulated Gene Expression in Arabidopsis thaliana. Molecular Plant 7: 1303-1315.

Wu, F.-H., Shen, S.-C., Lee, L.-Y., Lee, S.-H., Chan, M.-T., and Lin, C.-S. (2009). Tape-Arabidopsis Sandwich - a simpler Arabidopsis protoplast isolation method. Plant Methods 5: 16.

Xiong, F., Zhang, R., Meng, Z., Deng, K., Que, Y., Zhuo, F., Feng, L., Guo, S., Datla, R., and Ren, M. (2017). Brassinosteriod Insensitive 2 (BIN2) acts as a downstream effector of the Target of Rapamycin (TOR) signaling pathway to regulate photoautotrophic growth in Arabidopsis. New Phytologist 213: 233-249.

Xiong, Y., Contento, A.L., Nguyen, P.Q., and Bassham, D.C. (2007). Degradation of Oxidized Proteins by Autophagy during Oxidative Stress in Arabidopsis. Plant Physiology 143: 291-299.

Xiong, Y., McCormack, M., Li, L., Hall, Q., Xiang, C., and Sheen, J. (2013). Glucose-TOR signalling reprograms the transcriptome and activates meristems. Nature 496: 181-186.

Xiong, Y. and Sheen, J. (2014). The role of target of rapamycin signaling networks in plant growth and metabolism. Plant physiology 164: 499-512.

Yan, Z., Zhao, J., Peng, P., Chihara, R.K., and Li, J. (2009). BIN2 functions redundantly with other Arabidopsis GSK3-like kinases to regulate brassinosteroid signaling. Plant physiology 150: 710-21.

Yang, H., Rudge, D.G., Koos, J.D., Vaidialingam, B., Yang, H.J., and Pavletich, N.P. (2013). MTOR kinase structure, mechanism and regulation. Nature 497: 217-223.

Yang, X., Srivastava, R., Howell, S.H., and Bassham, D.C. (2016). Activation of autophagy by unfolded proteins during endoplasmic reticulum stress. The Plant Journal 85: 83-95.

Ye, K., Li, H., Ding, Y., Shi, Y., Song, C., Gong, Z., and Yang, S. (2019). BRASSINOSTEROID-INSENSITIVE2 Negatively Regulates the Stability of Transcription Factor ICE1 in Response to Cold Stress in Arabidopsis. The Plant Cell 31: 2682-2696.

Yin, Y., Wang, Z.-Y., Mora-Garcia, S., Li, J., Yoshida, S., Asami, T., and Chory, J. (2002). BES1 Accumulates in the Nucleus in Response to Brassinosteroids to Regulate Gene Expression and Promote Stem Elongation. Cell 109: 181-191.

Youn, J.-H. and Kim, T.-W. (2015). Functional Insights of Plant GSK3-like Kinases: Multi-Taskers in Diverse Cellular Signal Transduction Pathways. Molecular Plant 8: 552-565.

Zhang, F., Wang, Y., Li, G., Tang, Y., Kramer, E.M., and Tadege, M. (2014). STENOFOLIA recruits TOPLESS to repress 
bioRxiv preprint doi: https://doi.org/10.1101/2021.02.12 431003. this version posted February 13,2021 . The copyright holder for this preprint (which was not certified by peer review) is the author/funder, who has granted bioRxiv a license to display the preprint in perpetuity. It is made available under aCC-BY-NC-ND 4.0 International license.

ASYMMETRIC LEAVES2 at the leaf margin and promote leaf blade outgrowth in Medicago truncatula. Plant Cell 26: 650-664. Zhang, Y., Song, G., Lal, N.K., Nagalakshmi, U., Li, Y., Zheng, W., Huang, P., Branon, T.C., Ting, A.Y., Walley, J.W., and Dinesh-Kumar, S.P. (2019). TurbolD-based proximity labeling reveals that UBR7 is a regulator of N NLR immune receptor-mediated immunity. Nature Communications 10: 3252.

Zhang, Z., Zhu, J.-Y., Roh, J., Marchive, C., Kim, S.-K., Meyer, C., Sun, Y., Wang, W., and Wang, Z.-Y. (2016). TOR Signaling Promotes Accumulation of BZR1 to Balance Growth with Carbon Availability in Arabidopsis. Current Biology 26: 1854-1860. 
bioRxiv preprint doi: https://doi.org/10.1101/2021.02.12.431003; this version posted February 13, 2021. The copyright holder for this preprint

a (which was not certified by peer review) is the author/funder, who has granted bioRxiv a license to display the preprint in perpetuity. It is made

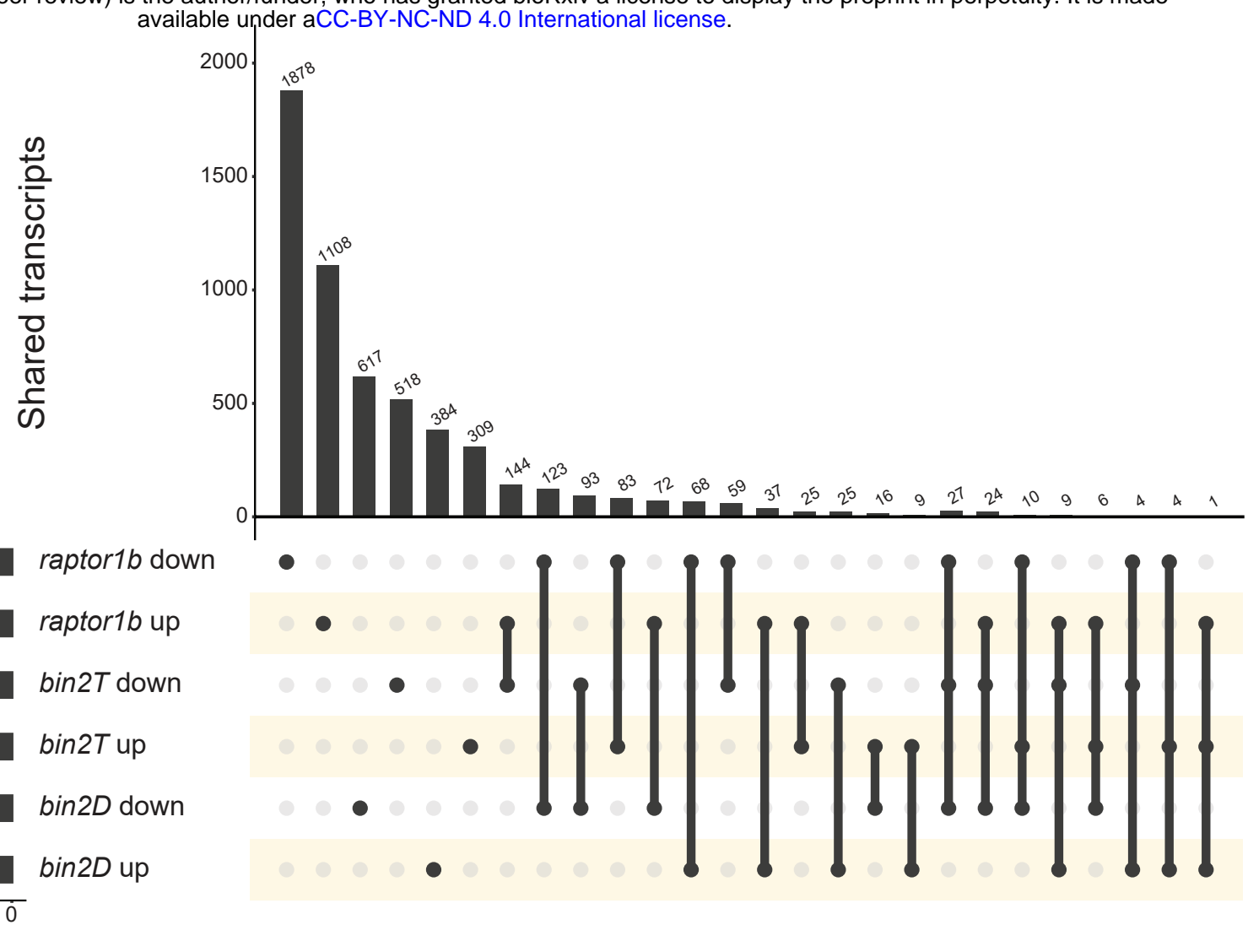

b

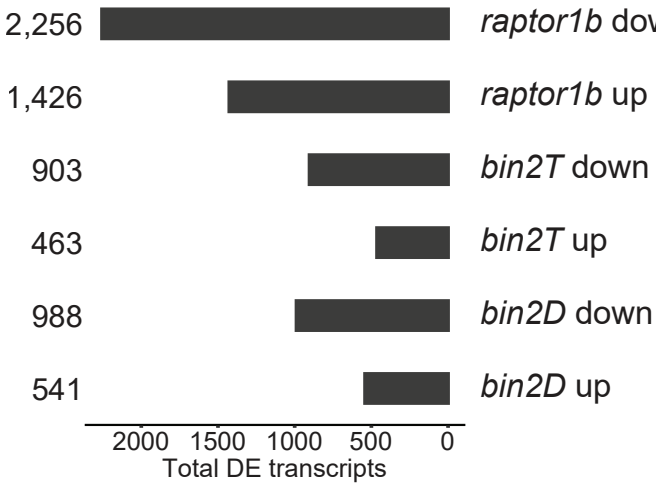

C
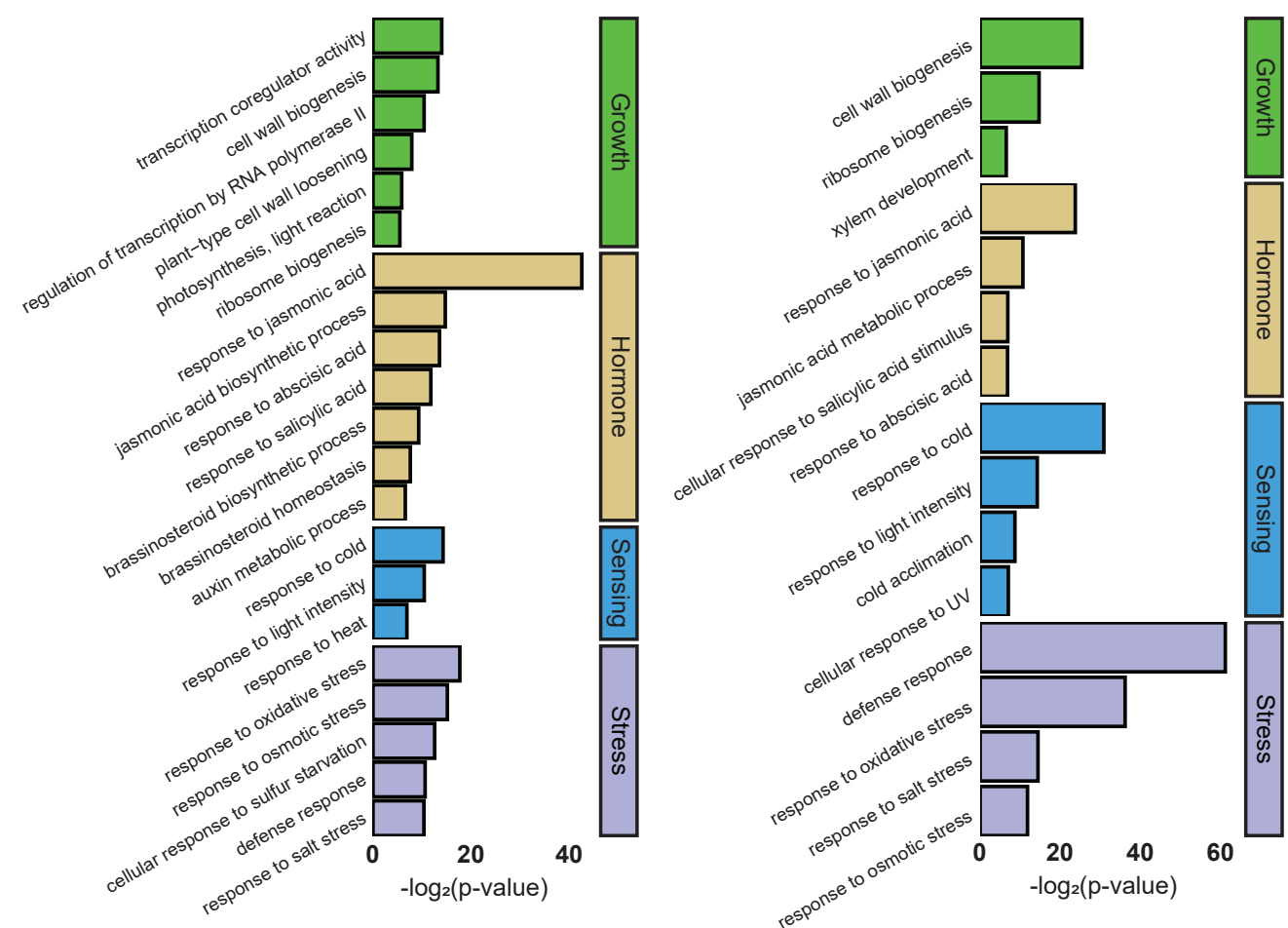

d

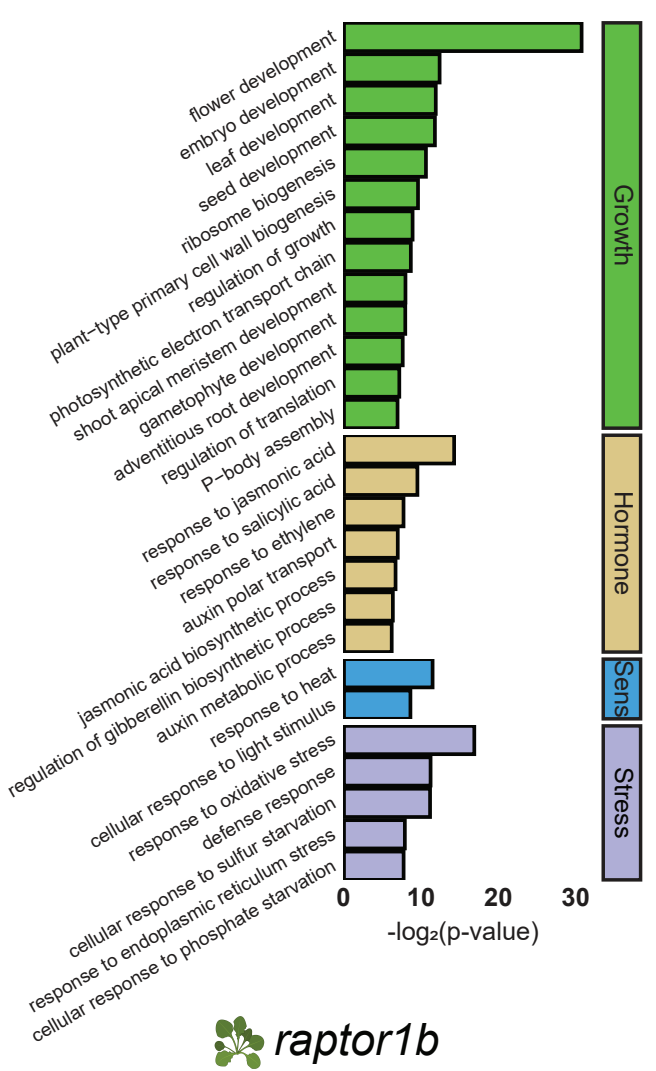

$\operatorname{bin} 2 D$

米=bin2T

Supplementary Fig. 1. Transcriptomic analysis of bin2 and raptor1b mutants. a, UpSet plot showing overlap of $D E$ transcripts between bin2D, bin $2 T$, and raptor $1 b$. b-d, Selection of significant GO biological processes among differential expressed transcripts on bin2D (b), bin2 $T$ (c), and raptor1b (d). Differential expression was defined as q-value $<0.05$ and absolute fold-change $>1.32$. 
bioRxiv preprint doi: https://doi.org/10.1101/2021.02.12.431003; this version posted February 13, 2021. The copyright holder for this preprint a (which was not certified by peer review) is the author/funder, who has granted bioRxiv a license to display the preprint in perpetuity. It is made

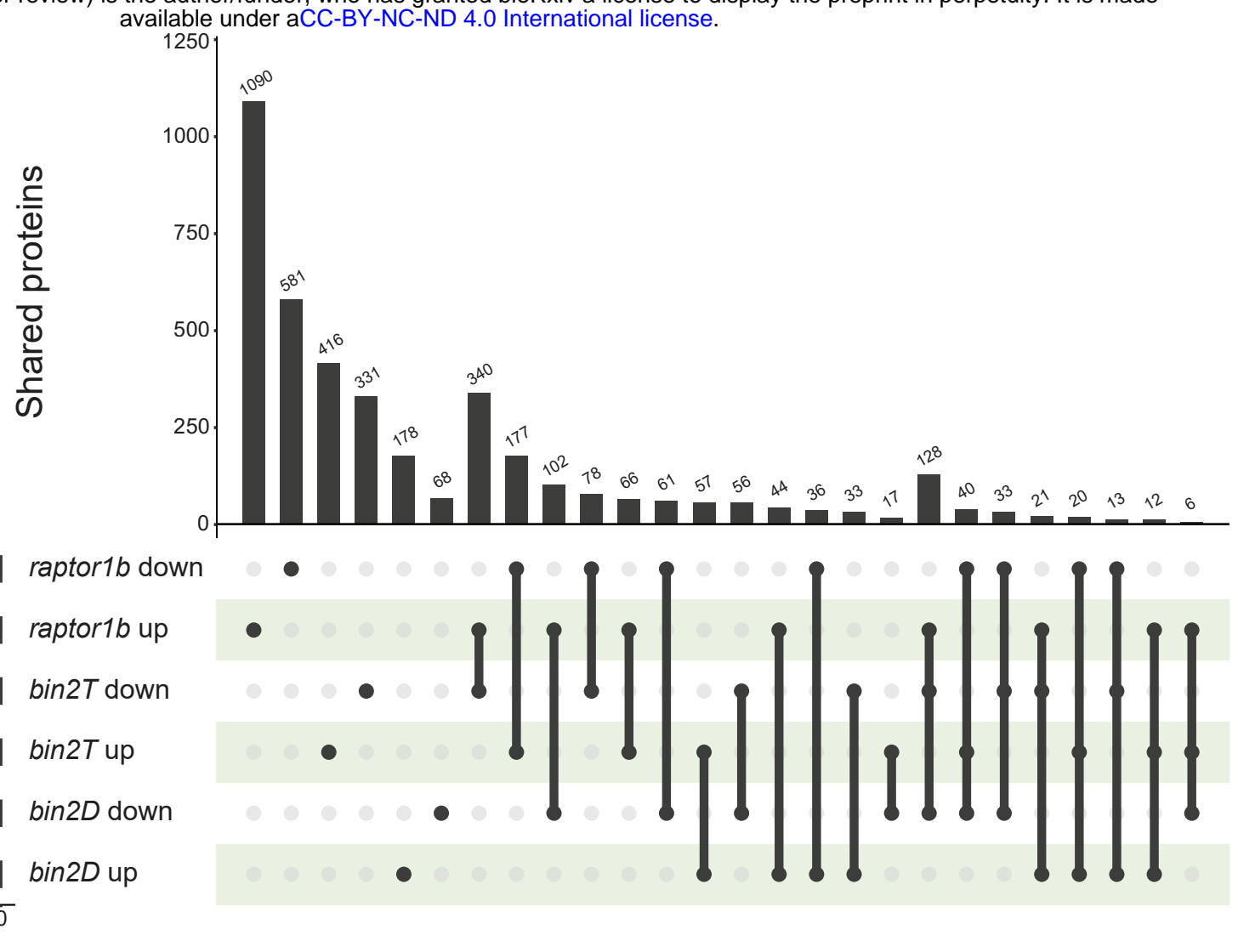

b

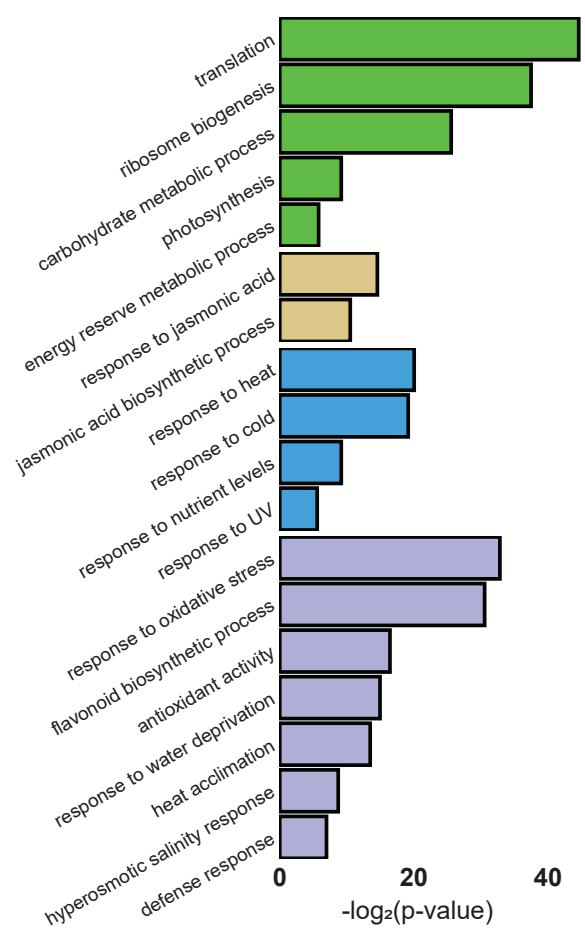

$\operatorname{bin} 2 D$
C

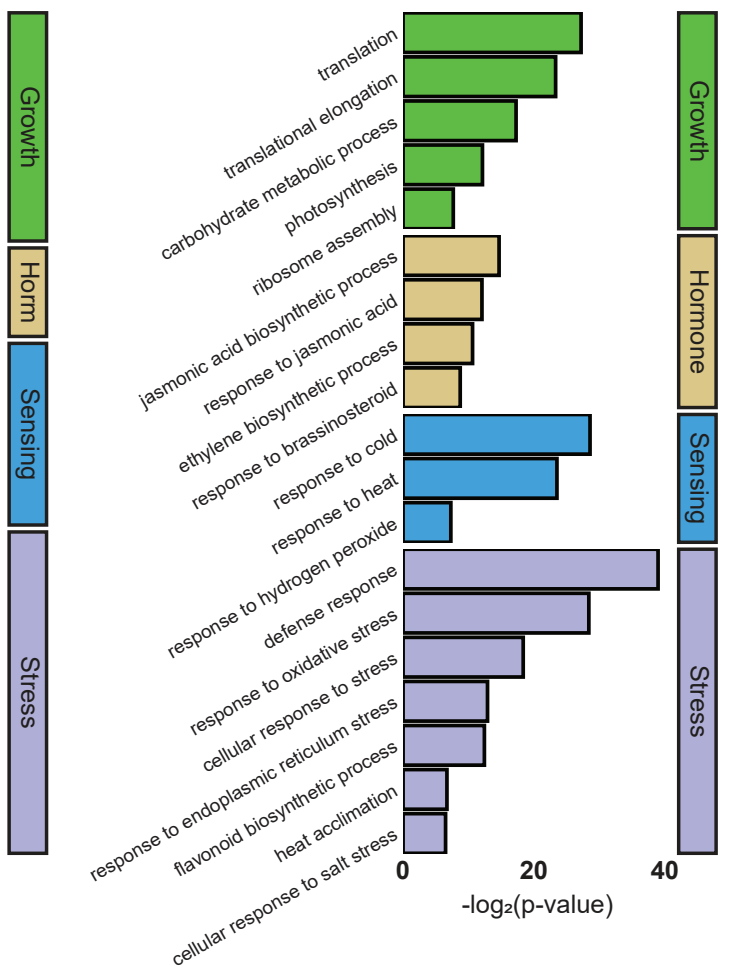

头 $=\operatorname{bin} 2 T$ d

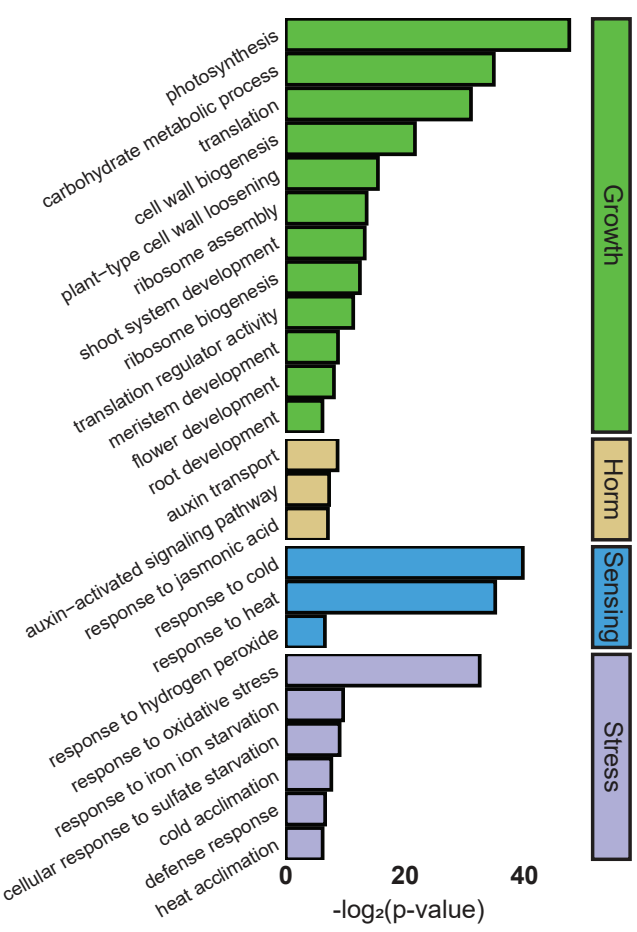

*aptor1b

Supplementary figure 2. Proteomic analysis of bin2 and raptor1b mutants. a, UpSet plot showing overlap of DE proteins between bin2D, bin $2 T$, and raptor $1 b$. b-d, Selection of significant GO biological processes among differential expressed proteins on bin2D (b), bin2 $T$ (c), and raptor1b (d). Differential expression was defined as q-value $<0.1$. 


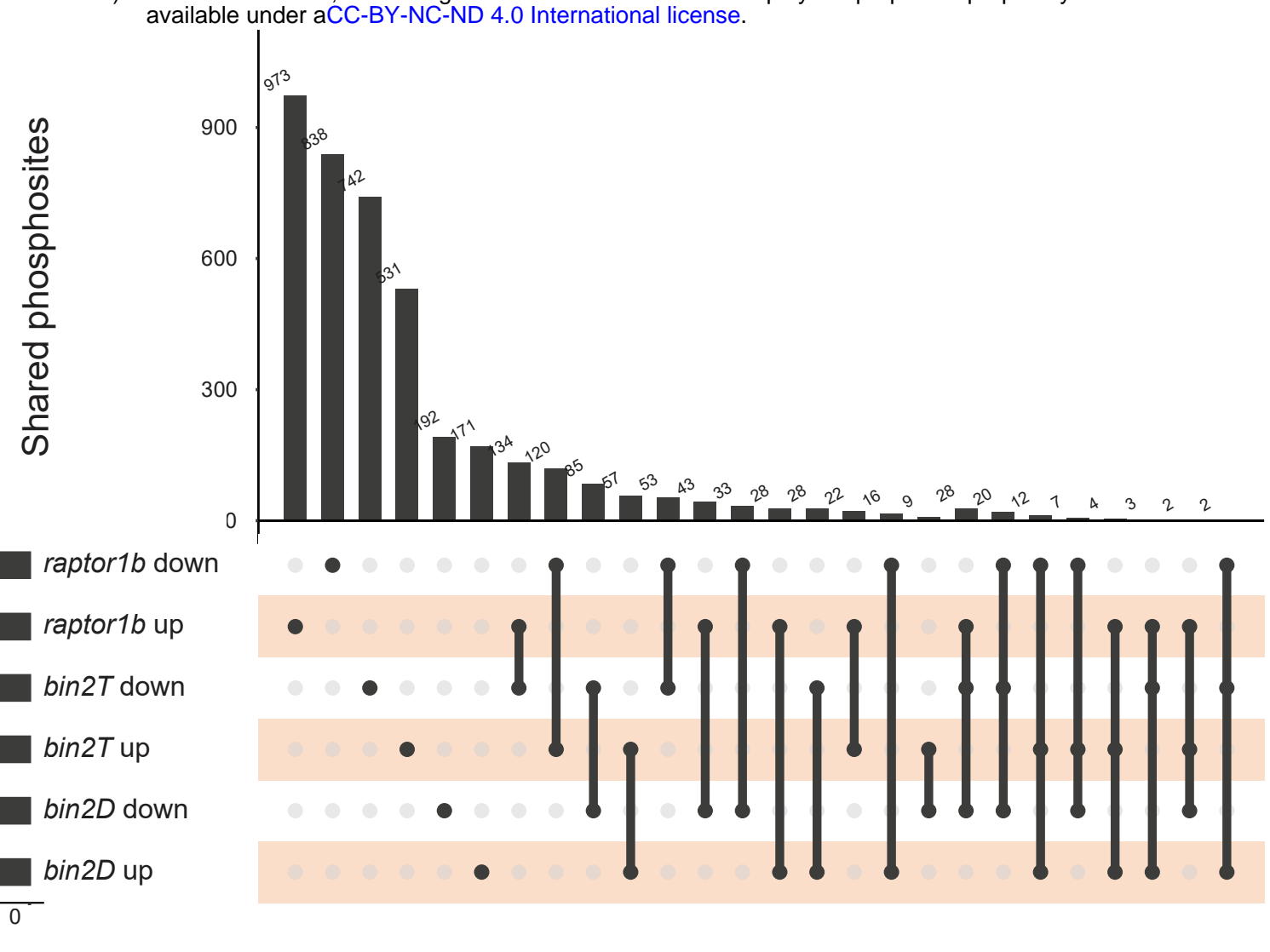

b

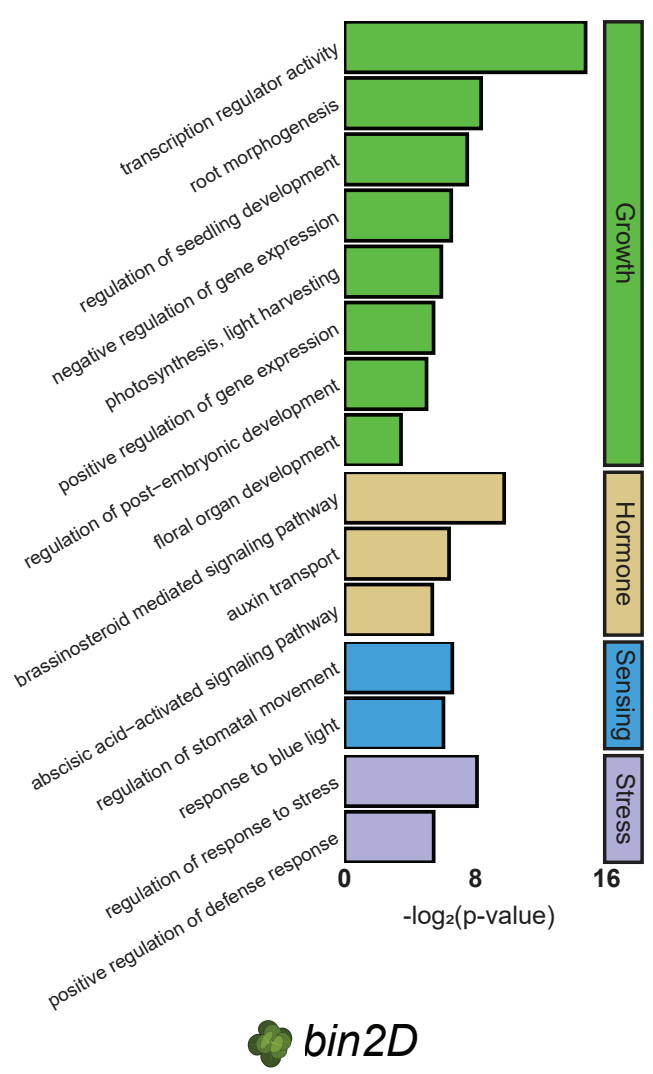

C

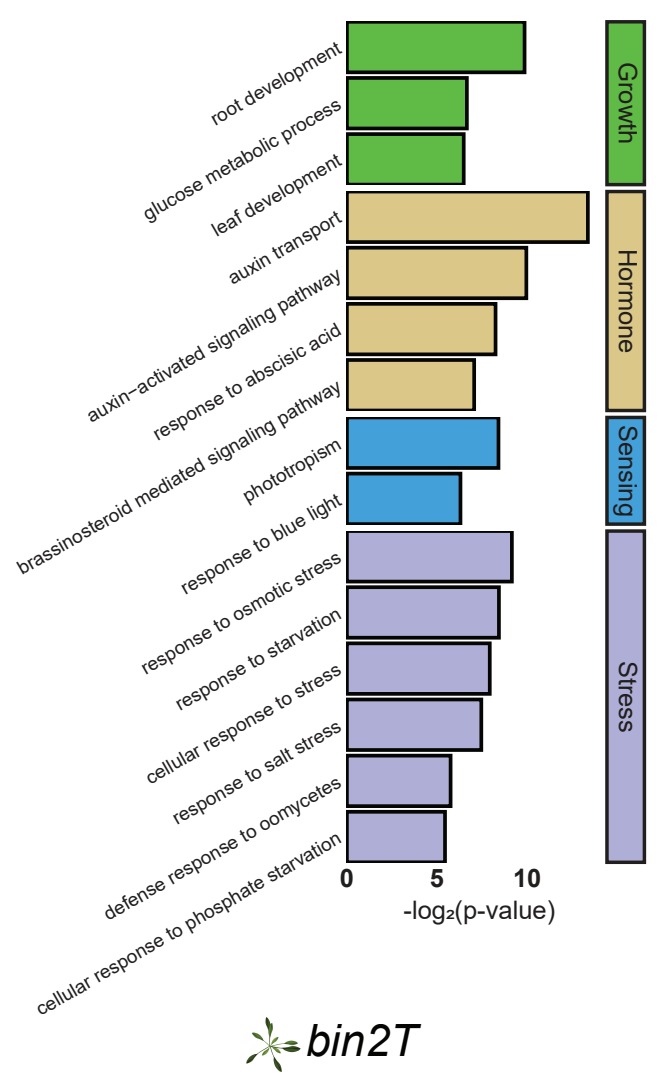

d

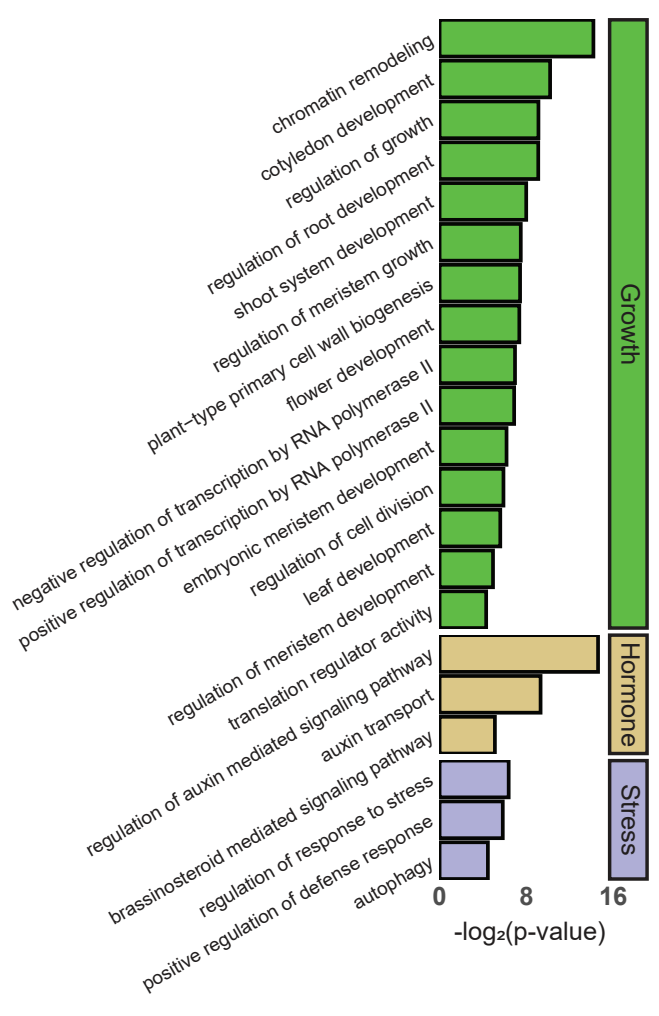

raptor1b

Supplementary figure 3. Phosphoproteomic analysis of bin2 and raptor1b mutants. a, UpSet plot showing overlap of differentially regulated phosphosites between bin2D, bin2T, and raptor $1 b$. b-d, Selection of significant $\mathrm{GO}$ biological processes among differentially upregulated phosphosites on bin2D (b), differentially downregulated phosphosites on bin2T (c), and differentially downregulated phosphosites on raptor1b (d). Differential phosphorylation was defined as q-value $<0.1$. 


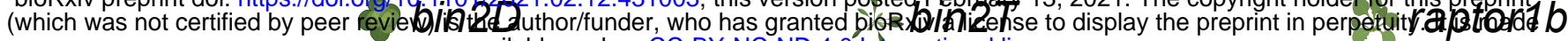
available under aCC-BY-NC-ND 4.0 International license.

Wang et al., 2014

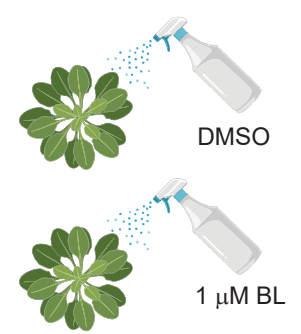

Overlap with dataset

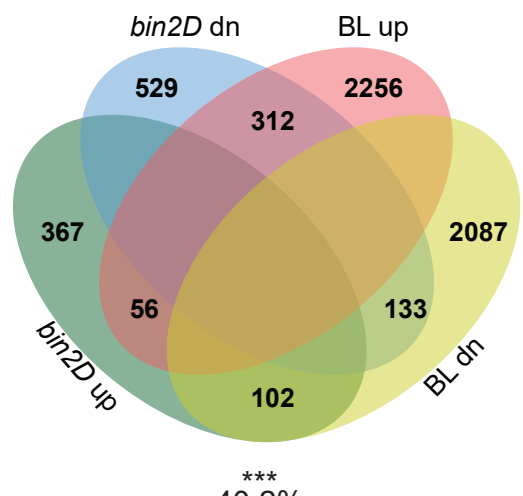

$40.2 \%$

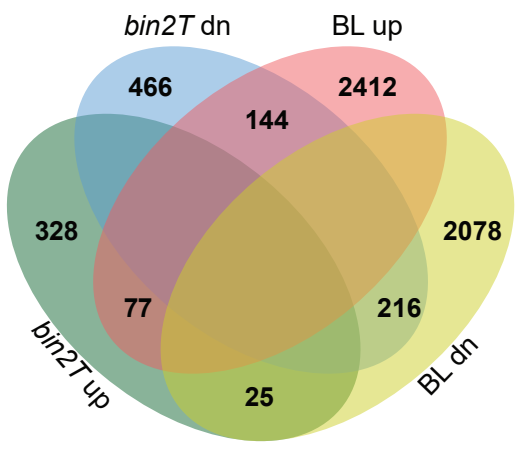

$36.8 \%$

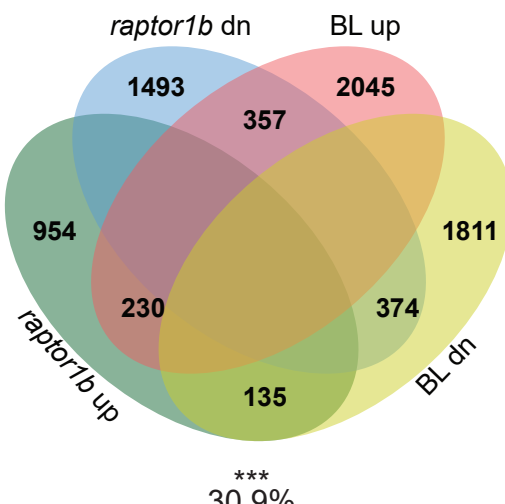

$30.9 \%$

b

Dong et al., 2015

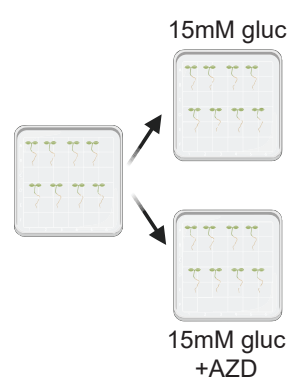

Overlap with dataset

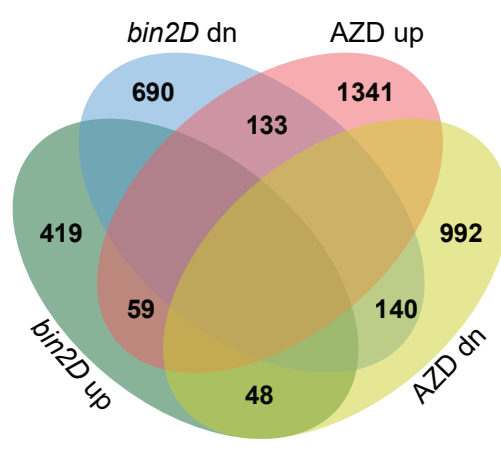

$25.5 \%$

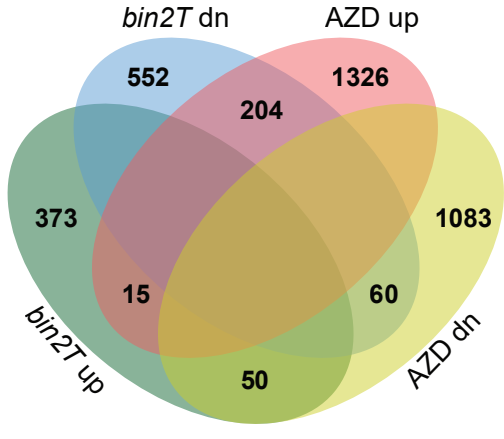

$26.2 \%$

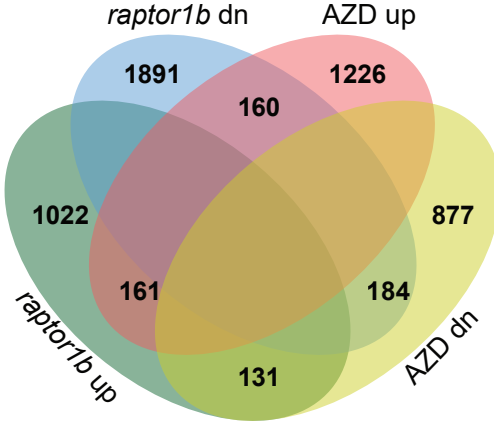

$17.9 \%$

Supplementary figure 4. Overlap with previously reported datasets. a,b, Overlap between $D E$ transcripts in bin2D, bin2 $T$, and raptor $1 b$ and BL-induced DE transcripts from Wang et al. (a) or AZD-induced DE transcripts from Dong et al. (b). Overlapping significance was assessed using hypergeometric test. ${ }^{* * *} p<0.001$ 
bioRxiv preprint doi: https://doi.org/10.1101/2021.02.12.431003; this version posted February 13, 2021. The copyright holder for this preprint (which was not certified by peer review) is the author/funder, who has granted bioRxiv a license to display the preprint in perpetuity. It is made TAIRavailable under aCC-BY-NC-ND 4.0 International license.

\section{annotation}

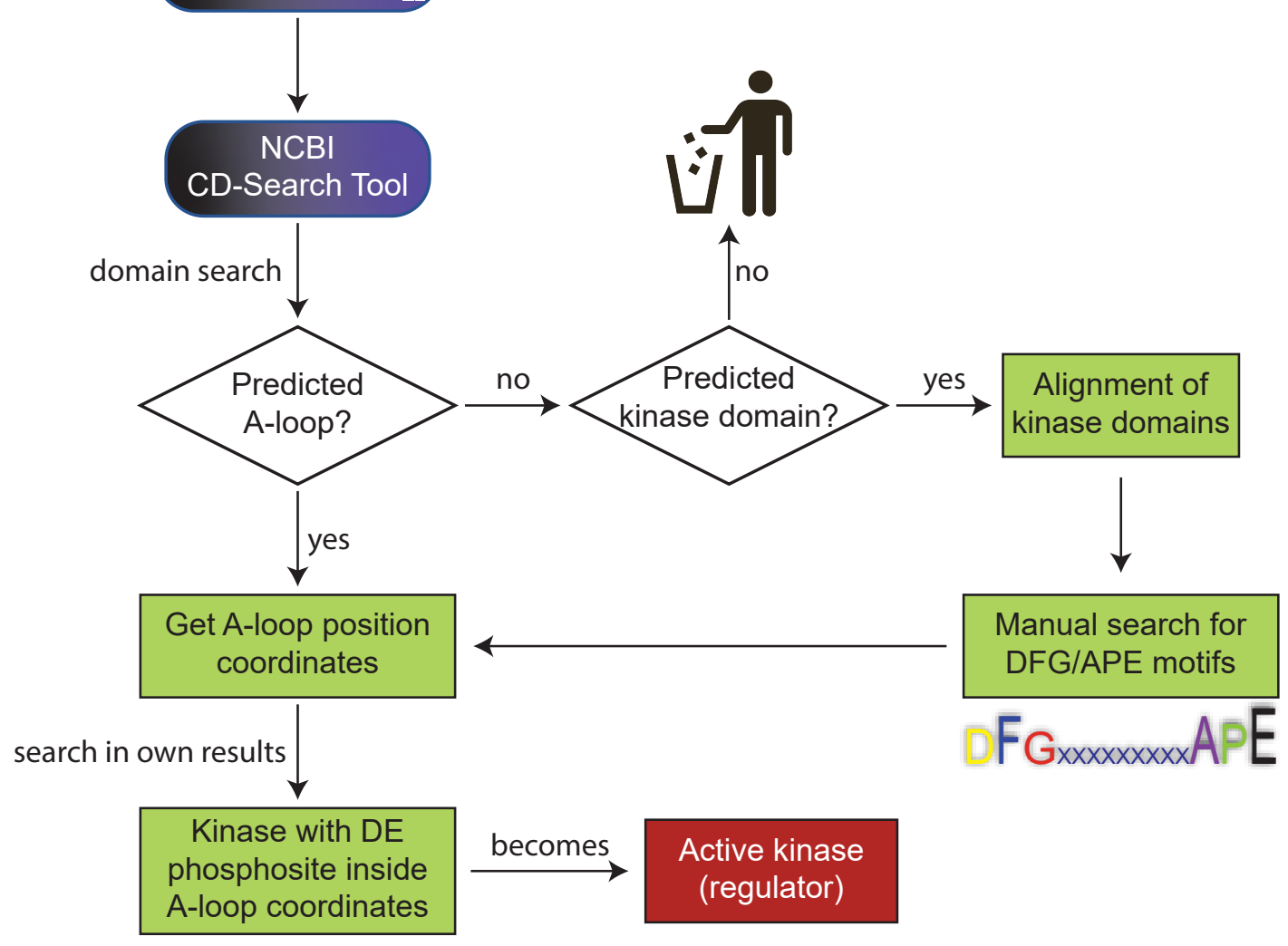

b

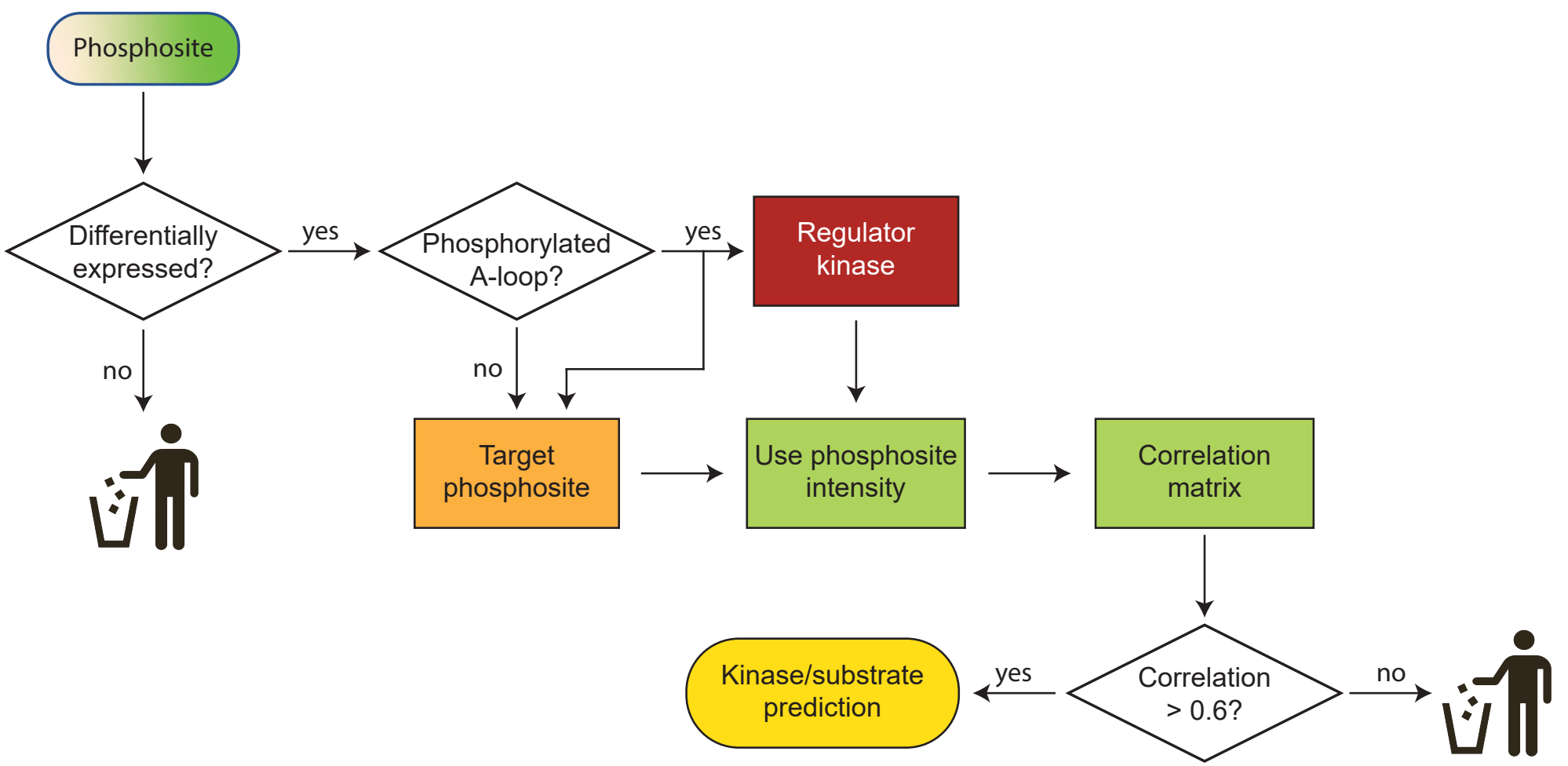

Supplementary figure $\mathbf{5}$. Workflow of signaling network reconstruction. a, Pipeline for activation loop coordinates annotation for Arabidopsis kinases. b, Logic diagram for kinase/substrate prediction. 
bioRxiv preprint doi: https://doi.org/10.1101/2021.02.12.431003; this versiopposted February 13, 2021. The copyright holder for this preprint (which was not certified by peer review) is the author/funder, who has granted bioRxiv a license to display the preprint in perpetuity. It is made available under aCC-BY-NC-ND 4.0 International license.

bin2D and raptor1b $\log _{2}(\mathrm{FC})$ phosphosite intensity

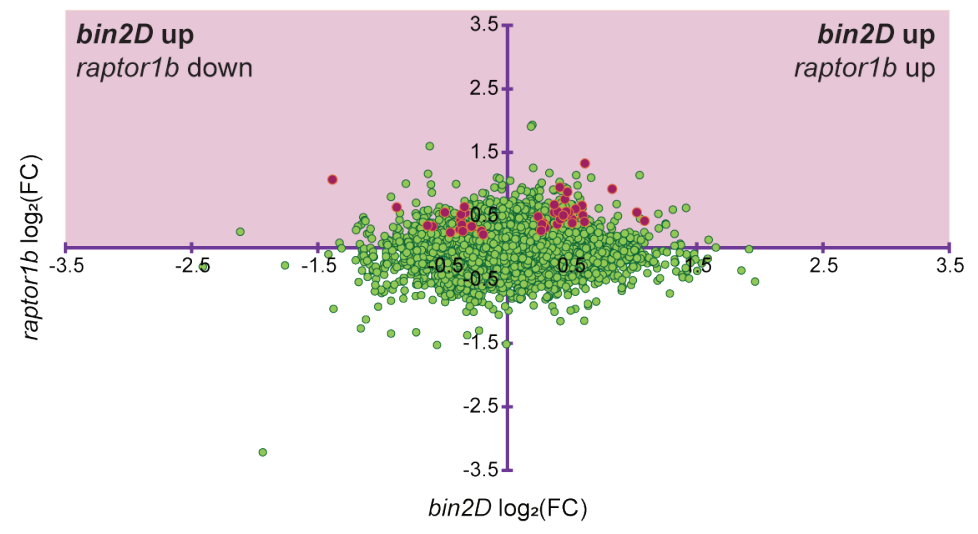

bin2T and raptor1b $\log _{2}(\mathrm{FC})$ phosphosite intensity

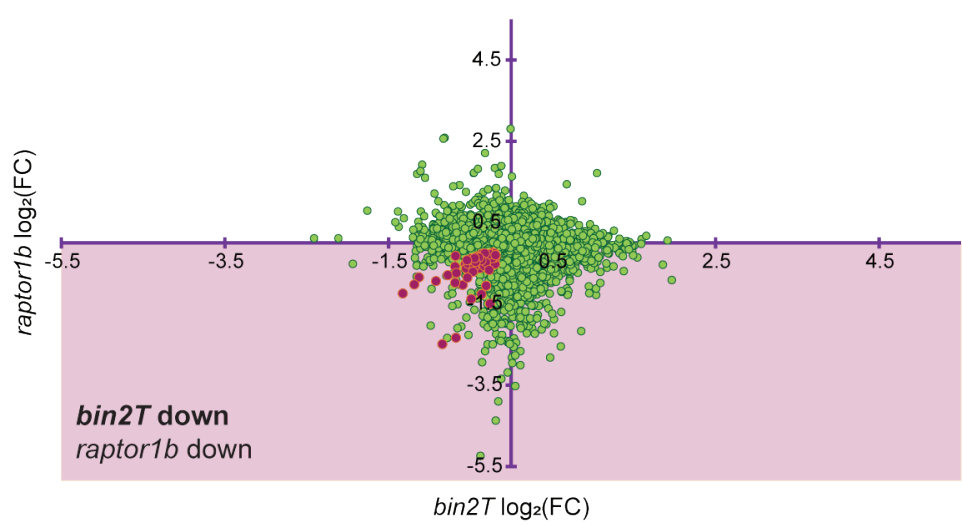

Candidate selection area

Candidate gene

Supplementary figure 6. Phosphosite intensity fold-change scatterplots and selection of candidate genes. a,b, Scatterplot showing phosphosite intensity $\log _{2}$ fold-change (mutant/WT) for raptor1b on the $\mathrm{x}$-axis and bin2D (a) or bin2T (b) on the y-axis. Red dots indicate phosphosites from selected candidate genes. 


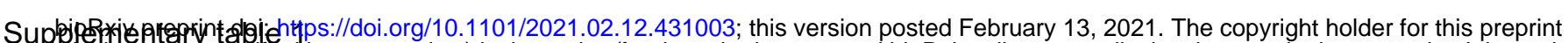
(which was not certified by peer review) is the author/funder, who has granted bioRxiv a license to display the preprint in perpetuity. It is made

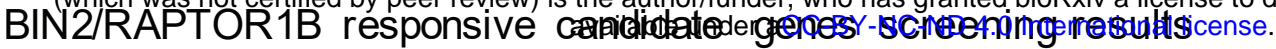

